\title{
Cell surface ectodomain integrity of a subset of functional HIV-1 envelopes is dependent on a conserved hydrophilic domain containing region in their $\mathrm{C}$-terminal tail
}

Sweety Samal ${ }^{1 \dagger}$, Supratik Das ${ }^{1 \dagger}$, Saikat Boliar ${ }^{1}$, Huma Qureshi ${ }^{1}$, Tripti Shrivastava', Naresh Kumar ${ }^{1}$, Sandeep Goswami ${ }^{1}$, Manish Bansal ${ }^{1}$ and Bimal K. Chakrabarti ${ }^{1,2,3^{*}}$

\begin{abstract}
Background: HIV-1 Env gp160 is cleaved to form gp120 and gp41 and the functional HIV-1 Env is a trimer of noncovalently associated heterodimeric subunits, gp120 and gp41. The cleaved, native, trimeric form of Envs expose only broadly neutralizing antibody (bNAb) epitopes while occluding epitopes targeted by non-neutralizing antibodies (non-NAbs). We and others have previously observed that efficient cleavage of Envs into their constituent subunits co-relates with specific binding to bNAbs and poor binding to non-neutralizing antibodies (non-NAbs). Such Envs have been identified from clades A, B and C which make up a majority of globally circulating HIV-1 strains. Frequently, the C-terminal tail (CT) of Envs is deleted to enhance expression and stabilize soluble Env-based vaccine immunogens. Deletion of CT of efficiently cleaved Indian clade C Env 4-2.J41 results in recognition by both NAbs and nonNAbs. It is to be noted that uncleaved Envs bind to both NAbs and non-NAbs. So we investigated whether altered antigenicity upon CT deletion of efficiently cleaved Envs is due to inefficient cleavage or conformational change as the mechanism by which the $C T$ regulates the ectodomain (ET) integrity is not well understood.

Results: We studied the effect of CT deletion in four membrane bound efficiently cleaved Envs, A5 (clade A), 4-2.J41 (clade C), JRFL and JRCSF (clade B). Deletion of CT of the Envs, JRCSF and 4-2.J41, but not JRFL and A5 alter their ET antigenicity/conformation without affecting the cleavage efficiency. We carried out a series of deletion mutation in order to determine the region of the CT required for restoring native-like antigenicity/conformation of the ET of 4-2. J41 and JRCSF. Extending the CT up to aa753 in 4-2.J41 and aa759 in JRCSF, which includes a conserved hydrophilic domain (CHD), restores native-like conformation of these Envs on the plasma membrane. However, CT-deletion in 4-2. J41 and JRCSF at the pseudovirus level has either no or only modest effect on neutralization potency.

Conclusion: Here, we report that the CHD in the CT of Env plays an important role in regulating the ET integrity of a subset of efficiently cleaved, functional Envs on the cell surface.
\end{abstract}

\footnotetext{
*Correspondence: bimal.chakrabarti@ablinc.com

${ }^{\dagger}$ Sweety Samal and Supratik Das have contributed equally to this work

${ }^{3}$ Innovation \& Translation, ABL, Inc., 9800 Medical Center Drive, Building

D, Rockville, MD 20850, USA

Full list of author information is available at the end of the article
} 


\section{Background}

The functional HIV-1 Env is a trimer of a non-covalently associated heterodimeric protein (gp120 and gp41 subunits), which mediates the entry of the virus into the host cell by interacting with cellular receptors [1-3]. The cleaved, native, trimeric form of Envs expose only broadly neutralizing antibody (bNAb) epitopes while occluding the epitopes targeted by non-neutralizing antibodies (non-NAbs) [4-7]. Since uncleaved and non-native forms of Env bind to non-NAbs [6-8] and may aid in immune evasion during HIV-1 infection by eliciting non-NAbs $[9,10]$, the current focus for designing immunogens is based on generating native, cleaved forms of Envs [6, 11, 12]. The only reported uncleaved, soluble Envs, that display exclusively bNAb epitopes, are the artificially generated single chain trimers [13-15]. However, in order to use Envs as immunogens for DNA, viral vector, virus-like particle based vaccination studies, it is essential that they are efficiently cleaved and display only broadly neutralizing epitopes on the cell surface. Furthermore, priming with Env expressing DNA followed by protein boosting has been shown to elicit more durable and qualitatively superior antibody response than protein alone [16-18]. Therefore, it is important that Envs need to display specifically bNAb epitopes on the cell surface when they are delivered through DNA. The American clade B Envs, JRFL and JRCSF, Indian clade C Env, 4-2.J41, and African clade A Envs, BG505 and A5 [19] are naturally occurring, efficiently cleaved Envs, when expressed on cell membrane and all of them bind exclusively to bNAbs [8, 20, 21]. Envs belonging to clades A, B and C make up about $75 \%$ of globally circulating HIV-1 strains.

The about 150 amino acid long CT (cytoplasmic tail) of Env contains several motifs [22-24] that are highly conserved across different HIV-1 clades and plays a critical role in modulating multiple Env functions $[25,26]$. The cytoplasmic tail contains the tyrosine (YXX $\phi)$ and di-leucine (LL) motifs, palmitoylated conserved cysteines, lentivirus lytic peptides (LLP), calmodulin binding domains [22-24]. The YXXФ (where X can be any amino acid, and $\Phi$ is a bulky, hydrophobic group) motif is shown to be crucial for their endocytosis following internalization of Env; thus disruption of this motif increases Env surface expression $[25,27]$. Therefore, CT of Env is regularly deleted in order to enhance expression [28] on cell surface and also to stabilize the soluble versions (SOSIP and NFL) of native-like trimeric Env immunogens [11, 14]. Thus, from a HIV-1 vaccine immunogen development point of view, it is important to understand the effect of $\mathrm{CT}$ truncation on antigenic properties of Env. Truncation of the CT affects ectodomain (ET) integrity of Envs but the cleavage status of these Envs is unknown [29-31]. In a recent report [32], using partially cleaved Envs, it has been shown that truncation of the C-terminus of these Envs expose non-neutralizing epitopes [32]. However, such studies have not been carried out in detail with naturally occurring, efficiently cleaved Envs, which are the closest approximations of functional HIV-1 Envs. Here, we show that naturally occurring, efficiently cleaved membrane-bound forms of Envs show phenotypic diversity in their ectodomain (ET) when their C-terminal tails are deleted without altering cleavage efficiency. C-terminally truncated JRFL and A5 Envs are able to maintain their antigenicity and conformation i.e. they do not expose non-neutralizing epitopes, but CT-deleted JRCSF and 4-2.J41 exposes non-neutralizing epitopes. Furthermore, CT deletion in all four Envs does not alter their cleavability. A conserved hydrophilic domain present in the CT of 4-2.J41 $\Delta \mathrm{CT}_{753}$ and JRCSF $\Delta \mathrm{CT}_{759}$ Envs restores native-like ET conformation similar to that of the wild type protein. CT-deletion in 4-2.J41 Env at the level of virus leads to modest resistance to conformational and trimer-selective bNAbs and sensitivity is not restored by restoring the conserved hydrophilic domain at the C-terminus. Furthermore, 4-2.J41 $\triangle \mathrm{CT}$ pseudoviruses become more sensitive to non-neutralizing antibodies at lower temperature and resistance is not restored by restoring the conserved hydrophilic domain. In addition, JRCSF $\triangle$ CT Env pseudotyped viruses show similar neutralization potency for bNAbs, except 10E8. The sensitivity of JRCSF $\triangle$ CT Env pseudotyped viruses to non-NAbs is similar to as it is seen for the virus pseudotyped with full length JRCSF Env. Taken together our findings demonstrate for the first time that conformational and antigenic integrity of the ectodomain of a subset of efficiently cleaved, functional Envs on the plasma membrane is dependent on the conserved hydrophilic domain present in their C-terminal tail. The significance of these findings on HIV-1 Env-based candidate vaccine development and the biology of these Envs are discussed.

\section{Results \\ C terminal tail deletion of 4-2.J41 affects ectodomain conformation/antigenicity}

It has been reported previously that truncation of the C-terminus of HIV-1 Envs generally leads to higher expression but affects their antigenicity, which is evident from exposure of epitopes for non-neutralizing antibodies [28-32]. These studies were primarily carried out with either partially cleaved Envs, or the cleavage efficiency of those Envs was not reported [30, 32]. However, studies with such Envs do not give a true representation of the structure-function relationship of the CT of functional Envs as an efficiently cleaved Env exposes only broadly neutralizing epitopes and are thus the closest representation of functional Envs, whereas uncleaved Envs bind 
to both neutralizing and non-neutralizing antibodies. The CT deletion in both 4-2.J41 and JRFL, have only modest effect on neutralization potency of pseudotyped viruses by bNAbs while they remain resistant to neutralization by non-NAbs $[5,20]$. However, deletion of the C-terminus of the Env, 4-2.J41 increases expression, but exposes non-neutralizing epitopes on the cell surface [20] suggesting that the effect of C-terminal truncation is different between Envs expressed on cell surface and on viral membrane. We, therefore, investigated the effect of C-terminal deletion on the cell surface expression, conformation and antigenicity of 4-2.J41 Env and determined the ratio of binding to bNAbs versus nonneutralizing antibodies (non-NAbs). As shown in Fig. 1a, C-terminal deletion up to position 711 eliminates all the major known domains of the CT of Env. Deletion of the C-terminus resulted in higher expression of 4-2.J41 $\Delta \mathrm{CT}$ compared to wild type 4-2.J41 as the cleavage-independent bNAb VRC01 showed much higher binding to the $\triangle \mathrm{CT}$ form as compared to wild-type (Fig. 1b) and we also observed enhanced binding to the non-NAbs F105, b6 and 17b (Fig. 1b). We determined the ratio of binding to different non-NAbs in comparison to VRC01 at $20 \mu \mathrm{g} /$ $\mathrm{ml}$ antibody concentration (Additional file 1: Table S1a). We find that the ratio of binding to non-NAbs F105, b6 and $17 \mathrm{~b}$ versus VRC01 changes from $0.22,0.38,0.47$ for wild type $4-2 . J 41$ to $0.64,0.73,0.71$ for $4-2 . J 41 \Delta C T$ (Additional file 1: Table S1a), respectively, suggesting that the non-neutralizing epitopes become exposed in 4-2.J41 $\Delta \mathrm{CT}$. We next tested the binding of 4-2.J41 and 4-2.J41 $\triangle \mathrm{CT}$ to the glycan-dependent, conformational bNAbs PG9, PG16, PGT121 and PGT128 in comparison to VRC01 and determined their binding ratios (Fig. 1c and Additional file 1: Table 1b). The ratio of binding to PG9 versus VRC01 did not change between 4-2.J41 and 4-2.J41 $\triangle$ CT but binding to PG16, PGT121 and PGT128 showed a decline from $0.7,1.68,1.48$ for 4-2.J41 to 0.44 , 1.22, 1.05, respectively, for 4-2.J41 $\Delta \mathrm{CT}$ (Fig. 1c and Additional file 1: Table S1b) suggesting that the conformation of 4-2.J41 is affected upon C-terminal tail deletion. Next, we tested 4-2.J41 and 4-2.J41 $\Delta C T$ for their ability to bind to the trimer-selective, cleavage-specific bNAb PGT151 in comparison to VRC01 and determined the ratio of binding (Fig. 1d and Additional file 1: Table S1c). The binding ratio fell from 0.71 for 4-2.J41 to 0.33 for 4-2. $\mathrm{J} 41 \Delta \mathrm{CT}$ (Additional file 1: Table S1c) suggesting that the native conformation of 4-2.J41 is affected upon CT deletion. Taken together these studies suggest that deletion of the C-terminal tail of 4-2.J41 cause conformational changes in 4-2.J41 $\Delta \mathrm{CT}$ and exposes non-neutralizing epitopes.

\section{C-terminal tail deleted 4-2.J41 is efficiently cleaved}

We next investigated whether the observed alteration in conformation/antigenicity of 4-2.J41 $\Delta \mathrm{CT}$ was due to inefficient cleavage of the $\Delta \mathrm{CT}$ version as uncleaved Envs bind to non-NAbs [8, 20, 21, 33]. We carried out neutravidin-agarose pull down of biotinylated cell surface proteins from 4-2.J41 $1_{\mathrm{WT}}$ and 4-2.J41 $\Delta \mathrm{CT}_{711}$ transfected cells and then probed the precipitate in western blots with anti-clade $\mathrm{C}$ antibodies (Fig. 2a). The crude whole cell extract showed two distinct bands corresponding to gp120 and the uncleaved gp140/gp160 (4-2. $\left.\mathrm{J}_{41} \Delta \mathrm{CT}_{711} / 4-2 . J 41_{\mathrm{WT}}\right)$ (Fig. 2a, left panel). The neutravidin-agarose precipitates of both 4-2.J41 $1_{\mathrm{WT}}$ and 4-2. $\mathrm{J} 41 \Delta \mathrm{CT}_{711}$ extracts showed a single band corresponding to gp120 (Fig. 2a, right panel) suggesting that like 4-2.J41 $1_{\mathrm{WT}}$ the CT deleted 4-2.J41, 4-2.J41 $\Delta \mathrm{CT}_{711}$ is efficiently cleaved on the cell surface. We further confirmed efficient cleavage of 4-2.J41 $\Delta \mathrm{CT}_{711}$ by gp120 shedding assay in the absence and presence of sCD4 (Fig. 2b). We found by both western blot analysis (data not shown) and ELISA assay (Fig. 2b) that both 4-2.J41 $1_{\mathrm{WT}}$ and 4-2. $\mathrm{J} 41 \Delta \mathrm{CT}_{711}$ proportionately shed gp120 and this shedding is enhanced upon incubation with sCD4 at the concentration of $40 \mathrm{ug} / \mathrm{ml}$ (Fig. 2b). Finally we looked at binding to the trimer-selective, cleavage-specific bNAb PGT151 at $20 \mu \mathrm{g} / \mathrm{ml}$ concentration by FACS-based cell surface antibody binding assay (Fig. 2c). We find that both 4-2. $\mathrm{J} 41_{\mathrm{WT}}$ and 4-2.J41 $\Delta \mathrm{CT}_{711}$ bind to PGT151 efficiently while the uncleaved 4-2.J41 $1_{\text {SEKS }}$ binds to PGT151 poorly (Fig. 2c). Taken together these studies demonstrate that the altered conformation/antigenicity of 4-2.J41 $\Delta \mathrm{CT}_{711}$ is not due to a defect in cleavage efficiency.

\section{LLP domains in 4-2.J41 Env CT do not restore ET conformation/antigenicity}

Having shown that 4-2.J41 $\Delta \mathrm{CT}_{711}$ Env has altered ectodomain antigenicity/conformation, we sought to examine whether any conserved domains in its CT are capable of restoring wild type antigenicity/conformation on cell surface. The highly conserved amphipathic $\alpha$-helical LLP1, LLP-2, LLP-3 domains located at the C-terminus of HIV-1 Env (CT) have been reported to play an important role in viral Env stability, oligomerization, membrane association and viral replication [34]. Hence, to investigate the importance of LLP domain of 4-2.J41 $1_{\mathrm{WT}}$ Env on antigenicity/conformation, we deleted LLP domains (LLP1: 828-856, LLP2: 768-785, LLP3: 785-815) in the CT of 4-2.J41 $1_{\mathrm{WT}}$ Env. We constructed four truncated forms of 4-2.J41 $1_{\mathrm{WT}}$ by incorporating stop codons at aa positions 717 (4-2.J41 $\left.\Delta \mathrm{CT}_{716}\right), 760$ (4-2.J41 $\left.\Delta \mathrm{CT}_{759}\right), 768$ $\left(4-2 . J 41 \Delta \mathrm{CT}_{767}\right)$ and $778\left(4-2.541 \Delta \mathrm{CT}_{777}\right)$, respectively (Fig. 3a). It is to be noted that the amino acid at position, 716 is located prior to the conserved domains in CT. In 


\section{a}

4-2.J41 $1_{W T}(g p 160)$

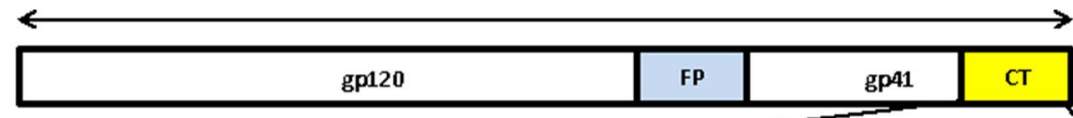

4-2.J1

$706 \leftarrow 724$

gp120 $768 \quad 785$

815

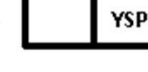

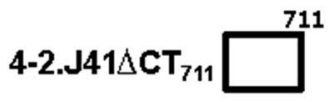

b

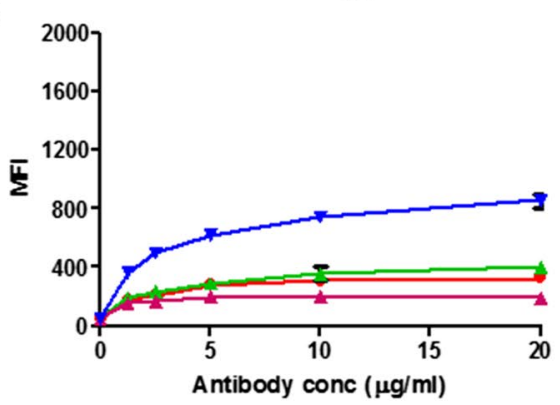

4-2.J41 $\mathrm{WT}$

C

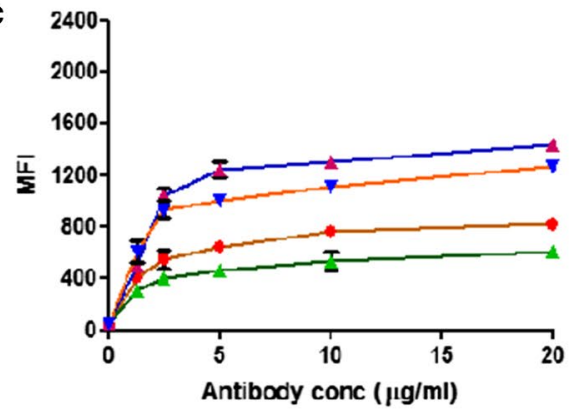

d

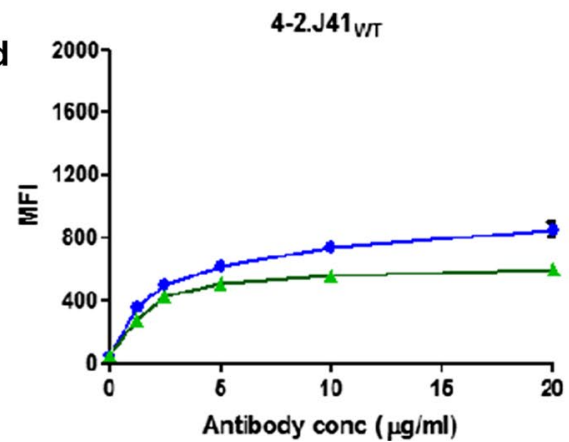

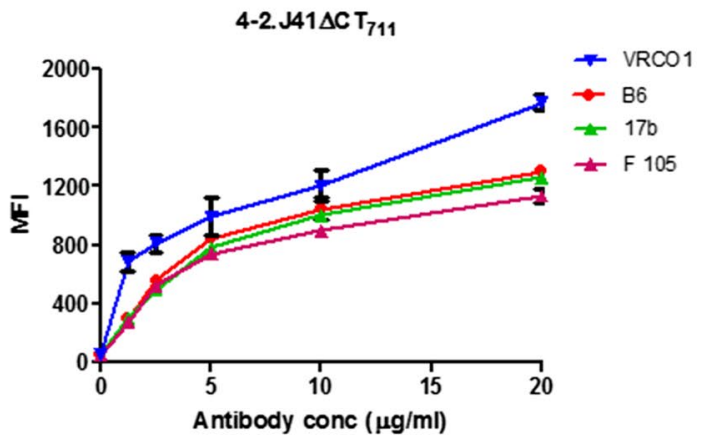

4-2.J41 $\Delta C \mathrm{~T}_{711}$

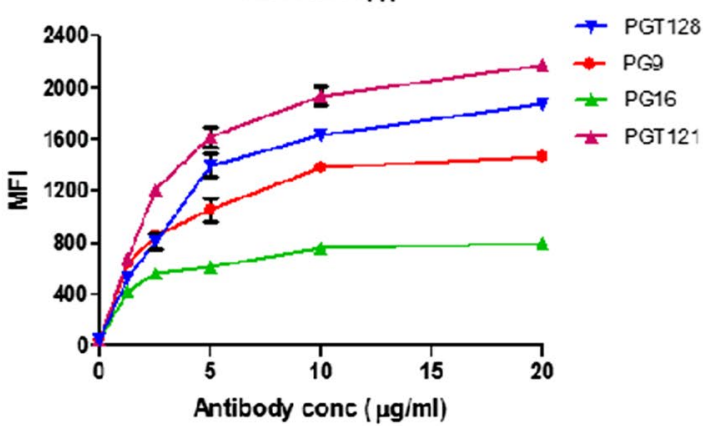

4-2.J41 $\Delta \mathrm{CT}_{711}$

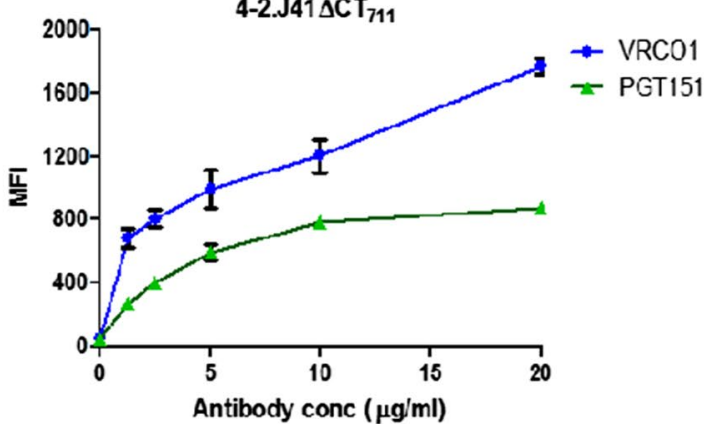

Fig. 1 CT-deleted 4-2.J41 Env has altered cell surface antigenicity/conformation. a Schematic of different domains in the C-terminal tail of HIV-1 Env. b FACS based cell surface staining assays of 4-2.J41 ${ }_{W T}$ and 4-2.J41 $\triangle C T_{711}$ with cleavage non-specific bNAb, VRC01 and non-NAbs, F105, 17b and b6 over a range of antibody concentrations. $\mathbf{c}$ FACS based cell surface staining assays of 4-2.J41 ${ }_{W T}$ and 4-2.J41 $\triangle C T_{711}$ with glycan-dependent, conformational bNAbs, PG9, PG16, PGT121, PGT128. d FACS based cell surface staining assays of 4-2.J41 WT $_{\text {and }}$ 4-2.J41 $\triangle C \mathrm{CT}_{711}$ with bNAb, VRC01 and trimer-selective, cleavage-specific bNAb, PGT151 
a

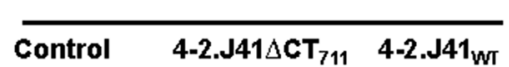
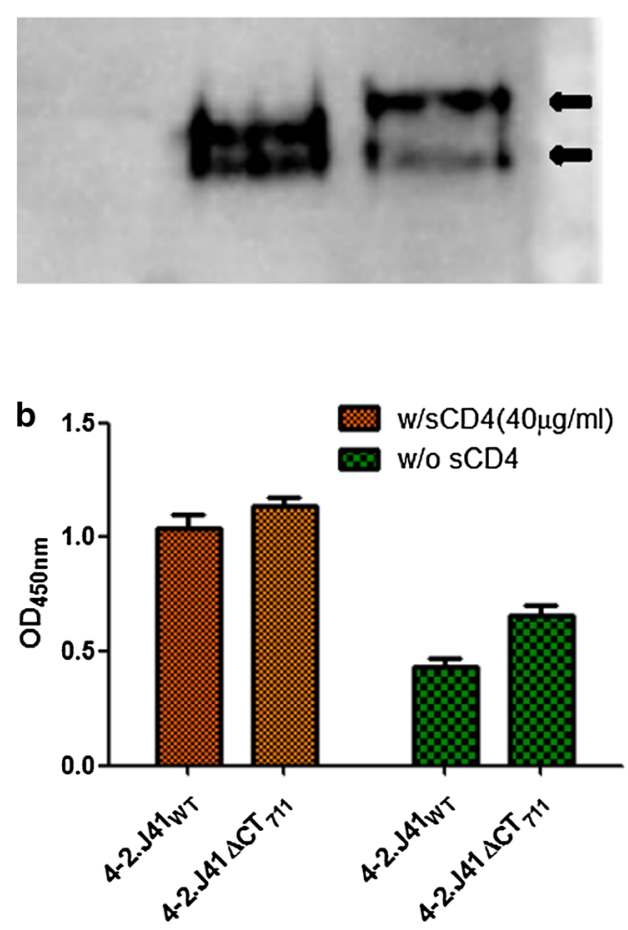

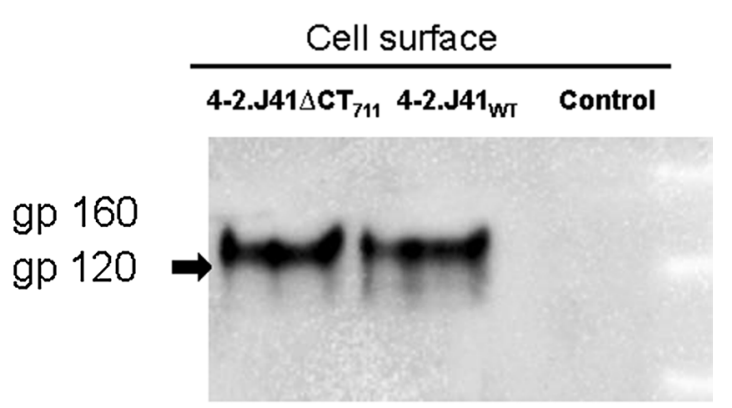

PGT $151(20 \mu \mathrm{g} / \mathrm{ml})$

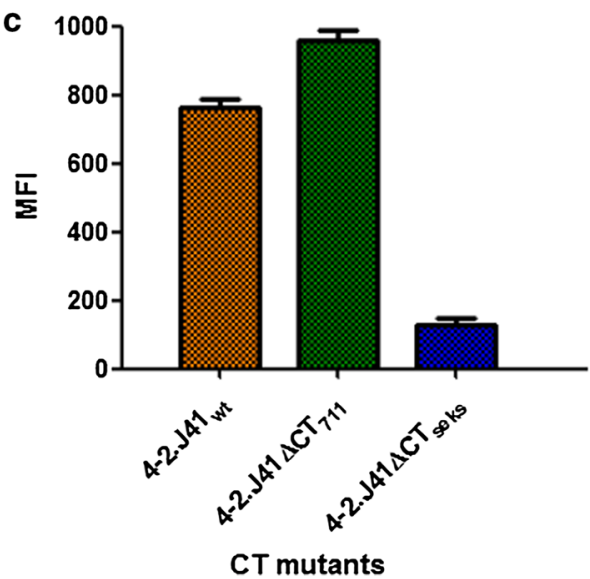

Fig. 2 CT-deleted 4-2.J41 Env is cleaved. a Western blot analysis of cell lysates (left panel) and neutravidin-agarose precipitates (right panel) of cell surface biotinylated mock transfected, 4-2.J41 $\mathrm{wT}_{\mathrm{W}}$ and 4-2.J41 $\triangle \mathrm{CT}_{711}$ Env transfected 293T cell lysates are shown. b Spontaneous and sCD4-induced gp120 shedding of 4-2.J41 $1_{W T}$ and 4-2.J41 $\triangle \mathrm{CT}_{711}$ Env transfected 293T cells incubated separately with $20 \mathrm{ug} / \mathrm{ml}$ of sCD4 determined by ELISA.

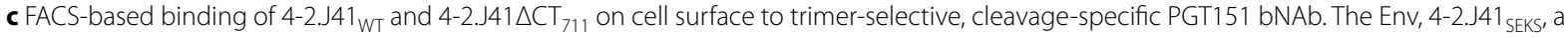
cleavage defective, non-native form of 4-2.J41 Env was used as a negative control. The error bars indicate the standard mean obtained from three independent experiments performed in duplicates, $P<0.0001$

4-2.J41 $\Delta \mathrm{CT}_{759}$ Env, the conserved aa, Cys at position, 764 is absent, which ablates the palmitoylation site. In 4-2.J41 $\Delta \mathrm{CT}_{767}$ Env, the conserved aa Cys at 764 is present. In 4-2.J41 $\Delta \mathrm{CT}_{777}$ Env, a small part of LLP2 domain is present. Like 4-2.J41 $1_{\mathrm{WT}} \mathrm{Env}$, the 4-2.J41 $\Delta \mathrm{CT}_{759}, 4-2$.

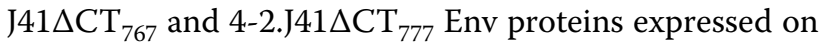
cell surface showed similar level of binding to VRCO1 and only minimal binding to non-NAb, F105 (Fig. 3b). In contrast, the 4-2.J41 $\Delta \mathrm{CT}_{716}$ Env, which retains only 10 amino acids of the cytoplasmic tail, showed increased binding to non-NAb F105 relative to VRC01 (Fig. 3b). Additionally, 4-2.J41 $\Delta \mathrm{CT}_{716}$ Env showed marked increase in binding to CD4bs-directed non-NAb, b6 and CD4i-directed non-NAb, 17b (Fig. 3c). Furthermore, reduction in binding to non-NAbs, b6 and $17 \mathrm{~b}$ was observed upon increasing the CT length beyond position 759 (Fig. 3c). Taken together, these results suggest that none of the LLP domains in 4-2.J41 $1_{\mathrm{WT}}$ Env are required for restoration of native ectodomain antigenicity/ conformation.

\section{Conserved hydrophilic domain in 4-2.J41 $\Delta \mathrm{CT}_{753}$ restores ET conformation/antigenicity}

Sequence analysis of HIV-1 Env proteins from different clades have revealed the presence of a conserved 22 amino acid domain (residues 724-745), different

Fig. 3 Restoration of cell surface antigenicity/conformation in CT mutants of 4-2.J41. a Schematic representation of LLP domain deleted mutants. $\mathbf{b}$ FACS-based cell surface staining assay of LLP domain deleted mutants with the bNAb, VRCO1 and non-NAb, F105. c Binding of LLP domain deleted mutants to CD4bs-directed non-NAb, b6 and CD4i-directed non-NAb, 17b at $20 \mu \mathrm{g} / \mathrm{ml}$ concentration. The error bars indicate the standard mean obtained from two independent experiments in duplicates. $P<0.0001$ 
a

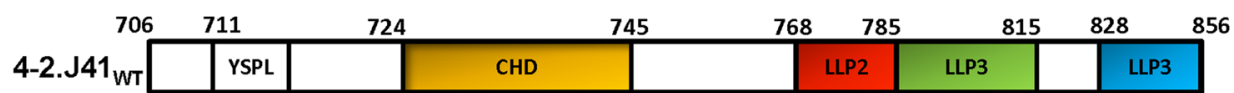

4-2.J41 $\Delta \mathrm{CT}_{716}$\begin{tabular}{|l|l|l|}
\hline & YSPL & \\
\hline
\end{tabular}

4-2.J41 $\Delta \mathrm{CT}_{759}$\begin{tabular}{|l|l|l|l|l|}
\hline & YSPL & & CHD & \\
\hline
\end{tabular}

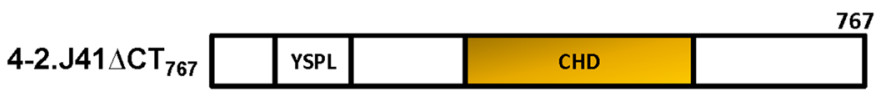
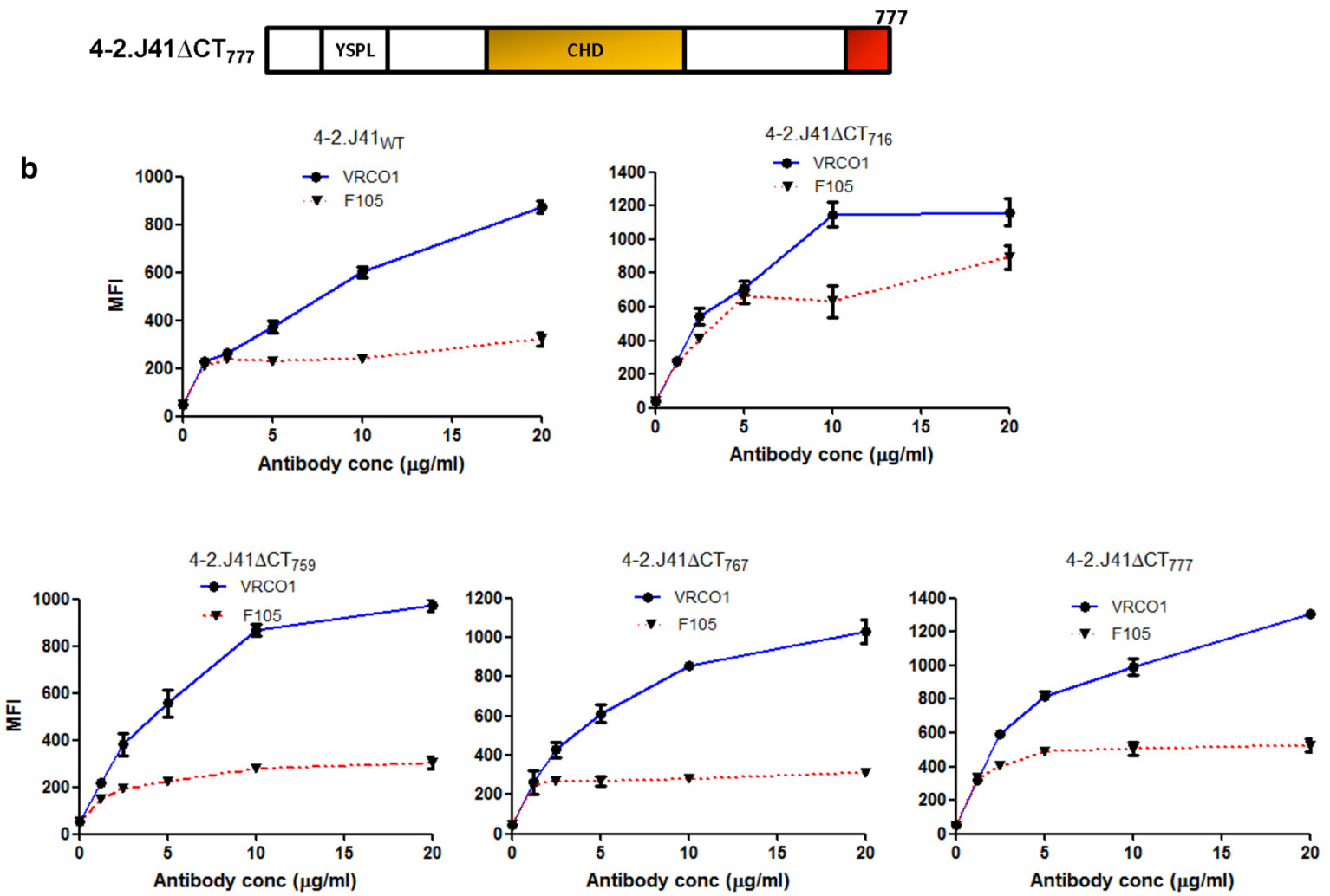

C

b6 $(20 \mu \mathrm{g} / \mathrm{ml})$
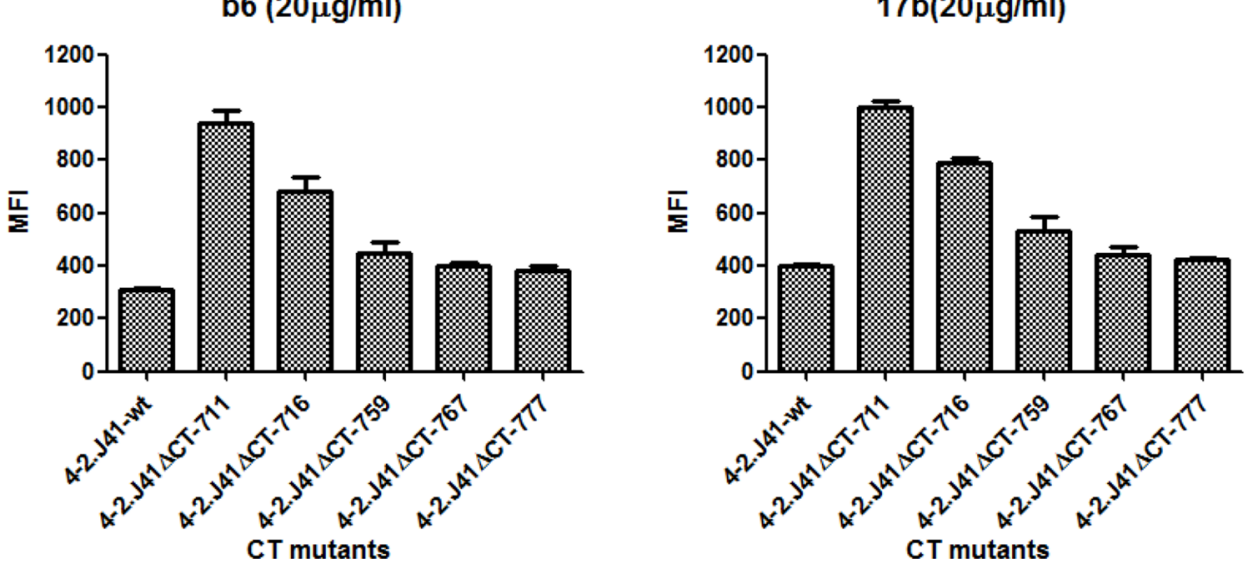
from LLP, in the CT of the HIV-1 Envs [22, 24]. The HIV-1 clade C Env, 4-2.J41 also harbors this conserved sequence (Figs. 1a, 3a, 4a). Previously, several studies described this conserved motif as hydrophilic and highly immunogenic $[35,36]$. We verified it by Kyte and Doolittle analysis (data not shown). To investigate the role of this conserved hydrophilic domain (CHD) on the antigenicity/conformation of 4-2.J41 ectodomain, we constructed a series of CT mutants (Fig. 4a). Proteins expressed from these constructs retained their ability to bind to bNAb, VRCO1 similar to $4-2 . J 41_{\mathrm{WT}}$ Env (Fig. 4b). The mutant protein expressed from the construct, 4-2.J41 $\Delta \mathrm{CT}_{736}$, which contains 12 amino acids of CHD, showed enhanced binding to non-NAb, F105 (Fig. 4b) similar to that of 4-2.J41 $\Delta \mathrm{CT}_{711}$ and 4-2. $\mathrm{J}_{41} \Delta \mathrm{CT}_{716}$ Envs. The cytoplasmic tail truncated mutant Env, 4-2.J41 $\Delta \mathrm{CT}_{744}$ also showed moderate increase in binding to non-NAb, F105 (Fig. 4b). The mutants truncated at aa positions, 746, 747, 749, 752 and 754 showed minimal binding to F105, comparable to 4-2.J41 $1_{\mathrm{WT}}$ Env (Fig. 4b). In order to determine whether native conformation of the CHD mutant Env proteins is maintained, we also studied the binding of conformation dependent non-neutralizing antibodies, $\mathrm{b} 6$ and $17 \mathrm{~b}$ to these mutant Envs (Fig. 4c). Truncating the CT at positions 737 (4-2. $\left.\mathrm{J}_{41} \Delta \mathrm{CT}_{736}\right)$ and $745\left(4-2 . \mathrm{J}_{41} \Delta \mathrm{CT}_{744}\right)$ resulted in partial deletion of the CHD whereas truncations at positions 746 (4-2.J41 $\left.\Delta \mathrm{CT}_{745}\right), 747$ (4-2.J41 $\left.\Delta \mathrm{CT}_{746}\right)$, and 749 (4-2. $\left.\mathrm{J} 41 \Delta \mathrm{CT}_{748}\right)$ restored the CHD domain. Restoring the CT to amino acid 746 resulted in reduction in binding to the non-NAbs b6 and 17b comparable to wild type 4-2.J41 but best results were obtained with the construct 4-2. $\mathrm{J}_{41} \Delta \mathrm{CT}_{753}$ (Fig. 4c). Taken together, our results suggest that the presence of the entire CHD, which ends at position 745 of the CT of 4-2.J41 Env is sufficient for restoring the native-like ectodomain conformation in terms of differential binding to VRCO1 and F105 and marginal binding to non-NAbs, b6 and $17 \mathrm{~b}$, similar to the wild type protein.

\section{Ectodomain properties of 4-2.J41 $\Delta \mathrm{CT}_{753} \mathrm{Env}$}

We next sought to determine whether the CHD can restore trimerization property similar to 4-2. $\mathrm{J} 41_{\mathrm{WT}}$ Env. We determined the binding of different $\triangle C T$ mutant Envs to the cleavage-specific, trimerselective bNAb, PGT151 [37] at a concentration of $20 \mu \mathrm{g} / \mathrm{ml}$ (Fig. 5a). As shown in Fig. 5a, b, if binding to the cleavage-non-specific bNAb VRCO1 is taken as a measurement of expression of both cleaved and uncleaved Envs on the cell surface, CT truncated Envs show decreased binding to PGT151. The partial or complete deletion of $\mathrm{CHD}$ at positions 717 (4-2.J41 $\left.\Delta \mathrm{CT}_{716}\right), 737$ (4-2.J41 $\left.\Delta \mathrm{CT}_{736}\right)$ and 745 (4-2. $\left.\mathrm{J}_{41} \Delta \mathrm{CT}_{745}\right)$ resulted in the mutants showing a binding ratio (PGT151:VRCO1) of $0.41,0.42$ and 0.48 , respectively as compared to the ratio of 0.73 for full length 4-2.J41 (Fig. 5b). However, after restoring the complete $\mathrm{CHD}$, the binding ratio of Env 4-2.J41 $\Delta \mathrm{CT}_{753}$ increased to 0.68 (Fig. 5 b).

We further investigated the binding of 4-2.J41 $\Delta \mathrm{CT}_{753}$ Env to bNAbs PGT121, PG9, PG16, and gp41 specific mAbs, 4E10, 10E8 (Fig. 5c). The bNAbs, PGT121, PG9 and PG16 are $\mathrm{N}$-glycan dependent antibodies, of which PGT121 binds to monomeric gp120 and the others bind to quaternary epitopes. 4-2.J41 $\Delta \mathrm{CT}_{753}$ Env binds to PGT121, PG9, PG16 and 10E8 similar to the wild type protein (Fig. 5c). However, the binding of 4-2.J41 $\Delta \mathrm{CT}_{753}$ Env to 4E10 was minimal (Fig. 5c). Next, we examined the binding ability of 4-2.J41 $\triangle \mathrm{CT}_{753}$ Env to different nonNAbs (Fig. 5c). The non-NAbs, b6, 17b, 2.2B and 44752D binds poorly to $4-2 . J 41 \Delta \mathrm{CT}_{753}$ and 4-2.J41 $1_{\mathrm{WT}}$ Envs (Fig. 5c). Overall, these results demonstrate that restoring the CT of 4-2.J41 up to position 753 containing the CHD domain restores ectodomain antigenicity and conformation similar to that of 4-2.J41 $1_{\mathrm{WT}}$. We further compared the cleavability of 4-2.J41 $1_{\mathrm{WT}}$ and 4-2.J41 $\Delta \mathrm{CT}_{753}$ by studying the CD4 induced gp120 shedding of 4-2.J41 $\Delta \mathrm{CT}_{753}$ with full length 4-2.J41 Env in the absence and presence of $40 \mathrm{ug} / \mathrm{ml}$ concentration of sCD4 (Fig. $5 \mathrm{~d}$ ). We found by ELISA assay that the shedding of gp120 from both 4-2. $J 41_{\mathrm{WT}}$ and 4-2.J41 $\Delta \mathrm{CT}_{753}$ is comparable and this shedding is enhanced upon incubation with $\mathrm{sCD} 4$ at the concentration of $40 \mathrm{ug} / \mathrm{ml} \mathrm{CD} 4$ (Fig. $5 \mathrm{~d}$ ) suggesting that 4-2.

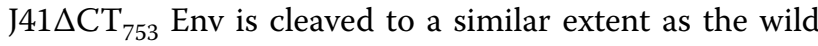
type protein.

\section{Effect of CT truncation on three other efficiently cleaved Envs}

Our data with 4-2.J41 Env clearly demonstrated that CT truncation alters ectodomain (ET) conformation/antigenicity which is restored similar to the wild type protein by restoring the conserved hydrophilic domain at its C-terminus. Next, we checked for ectodomain conformation/antigenic integrity upon CT truncation of the

(See figure on next page.)

Fig. 4 Minimal CT region of 4-2.J41 wt Env required for restoring native antigenicity/conformation. a Schematic representation of CHD deleted mutants of 4-2.J41 Env proteins. b FACS-based cell surface staining assay of CHD deleted mutants with the bNAb, VRCO1 and non-NAb, F105. c Binding of CHD deleted mutants to CD4bs-directed non-NAb, b6 and CD4i-directed non-NAb, 17b at $20 \mu \mathrm{g} / \mathrm{ml}$ concentration. The error bars indicate the standard mean obtained from two independent experiments run in duplicates $(P<0.0001)$ 
a

\begin{tabular}{|c|c|c|c|c|c|c|}
\hline 706 & 11 & 724 & & 785 & 815 & 828 \\
\hline $4-2 . J 41_{\mathrm{WT}}$ & YSPL & & CHD & P2 & LLP3 & LLP1 \\
\hline
\end{tabular}

4-2.J41 $\Delta \mathrm{CT}_{736} \quad$\begin{tabular}{|l|l|l|l|}
\hline & YSPL & & CHD \\
\hline
\end{tabular}

4-2.J41 $\Delta \mathrm{CT}_{744} \quad$\begin{tabular}{|l|l|l|l|}
\hline & YSPL & & CHD \\
\hline
\end{tabular}

4-2.J41 $\Delta \mathrm{CT}_{745}$\begin{tabular}{|l|l|l|l|}
\hline & YSPL & & CHD \\
\hline
\end{tabular}

4-2.J41 $\Delta \mathrm{CT}_{746} \quad$\begin{tabular}{|l|l|l|l|}
\hline & YSPL & & CHD \\
\hline
\end{tabular}

4-2.J41 $\Delta \mathrm{CT}_{748} \quad$\begin{tabular}{|l|l|l|l|l|}
\hline & YSPL & & CHD & \multicolumn{1}{|c|}{$\mathbf{7 4 8}$} \\
\hline
\end{tabular}

4-2.J41 $\Delta \mathrm{CT}_{751}$\begin{tabular}{|l|l|l|l|l|}
\hline & YSPL & & CHD & \multicolumn{1}{c|}{751} \\
\cline { 2 - 5 }
\end{tabular}

4-2.J41 $\Delta \mathrm{CT}_{753} \quad$\begin{tabular}{|l|l|l|l|l|}
\hline & YSPL & & CHD & \\
\hline
\end{tabular}

b
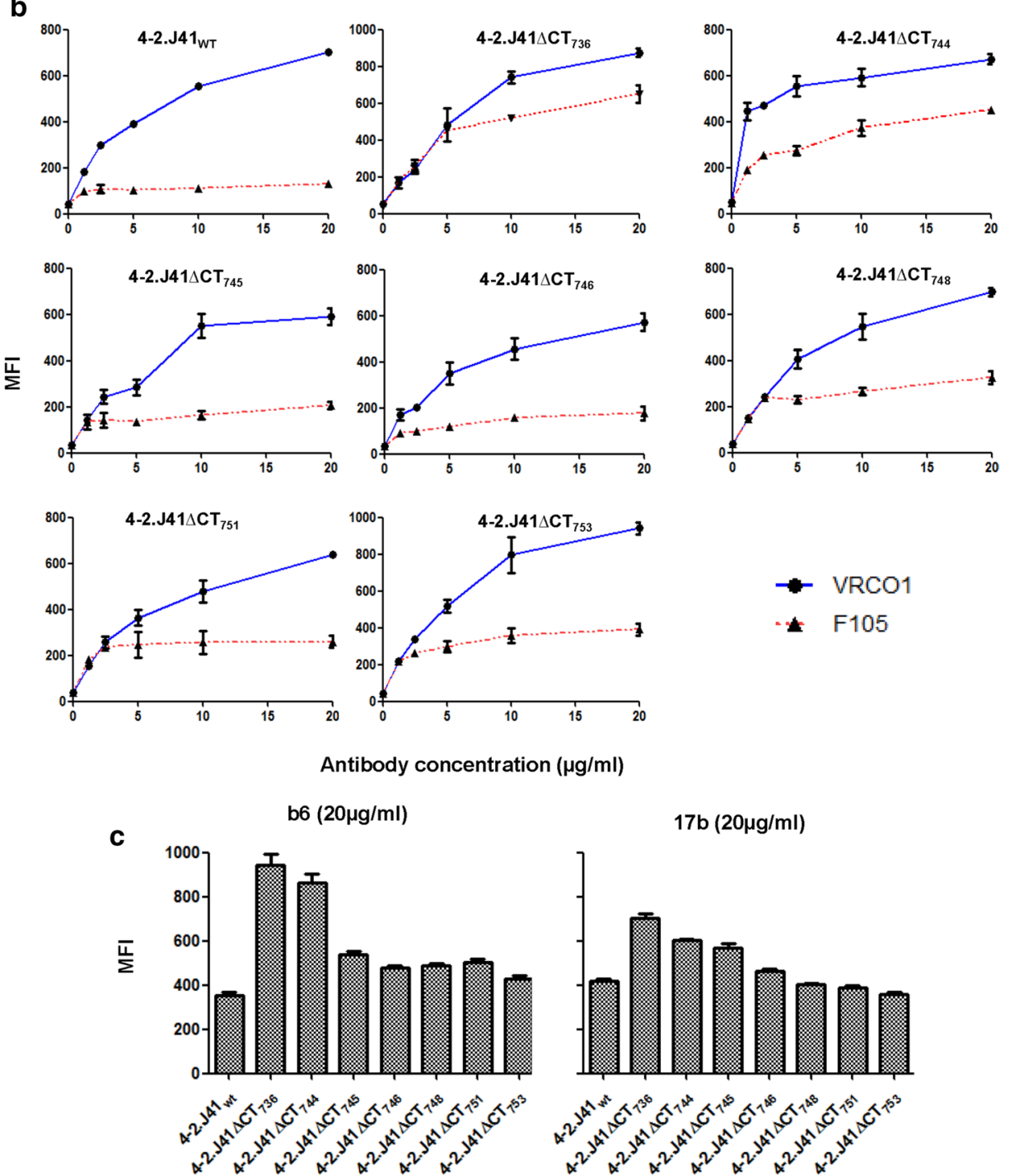

$17 \mathrm{~b}(20 \mu \mathrm{g} / \mathrm{ml})$

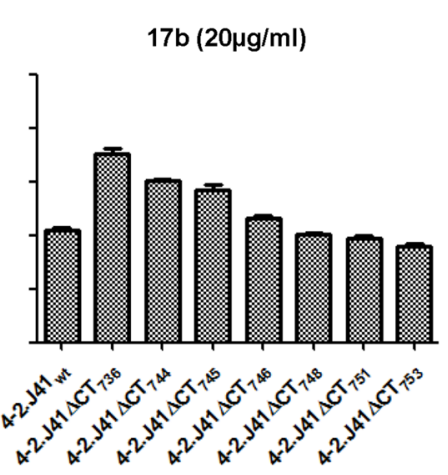

CT mutants 
(See figure on next page.)

Fig. 5 Restoration of conformation/antigenicity in 4-2.J41 ${ }_{753}$ Env. a Binding of CT-deleted mutant Envs expressed on 293 T cells to bNAbs, PGT151 (trimer-selective, cleavage-specific) and VRCO1 (cleavage non-specific) at $20 \mathrm{\mu g} / \mathrm{ml}$ antibody concentration by FACS based cell surface staining assay. The error bars indicate the standard mean obtained from two independent experiments run in duplicates, $P<0.0001$. b Ratio of binding to PGT151 versus VRC01 antibodies by different CT deleted mutants in cell-surface binding assay. $\mathbf{c}$ Binding of 4-2.J41 $\triangle \mathrm{CT}_{753}$ mutant (upper panel) to CD4bsdirected bNAb, VRC01, glycan directed, V1/V2 conformation-dependent bNAbs, PG9 and PG16; gp41-directed bNAbs, 10 E8 and 4E10 over a range of concentrations. Binding of 4-2.J41 $\triangle \mathrm{CT}_{753}$ mutant to non-NAbs, b6, 17b, 2.2B and 447-52D (lower panel). 4-2.J41 wT was used as positive control. d ELISA to determine the sCD4-induced gp120 shedding from 4-2.J41 $\triangle C_{753}$ Env transfected 293T cells incubated with and without sCD4 (40 $\left.\mu \mathrm{g} / \mathrm{ml}\right)$. 4-2.J41 $1_{W T}$ Env was used as positive control

efficiently cleaved clade B Envs, JRFL [8] and JRCSF [21] and the clade A Env, A5 [19]. We first investigated the effect of C-terminal deletion on the cell surface expression, conformation and antigenicity of JRFL, JRCSF, and A5. As shown in Fig. $6 \mathrm{a}-\mathrm{f}$, deletion of the C-terminus resulted in higher expression of JRFL $\triangle C T$ and JRCSF $\triangle C T$ as the cleavage-independent bNAbs b12, PG9 and PGT126 showed much higher binding to $\Delta C T$ forms as compared to wild-type but due to some unknown reasons, no difference in expression was observed between A5 and A5 $\triangle \mathrm{CT}$ as the cleavage-independent bNAbs, PG9 and PGT128 showed similar binding to both forms of Env (Fig. 6e). The major endocytosis signals GYXX $\phi$ and LL in the C-terminus of Env required for its internalization and whose removal or mutation increases Env expression on cell surface $[38,39]$ are both conserved in A5 (Additional file 2: Fig. S1a). Neither any toxicity nor any change in viability or toxicity induced changes in morphology of $293 \mathrm{~T}$ cells transfected with plasmid expressing A5 $\triangle \mathrm{CT}$ Env was observed (Additional file 2: Fig. S1b). JRCSF $\triangle$ CT Env binds to non-NAb F105 as efficiently as the bNAb, PGT126. Furthermore, the binding of JRCSF $\triangle$ CT Env to the conformational bNAb, PG9 is reduced to similar to the level of non-NAb, 412d. All these data suggest that the exposure of certain non-neutralizing epitopes in JRCSF $\triangle \mathrm{CT}$ is due to the conformational change after deletion of the CT domain of the Env (Fig. 6a). However, both JRFL $\Delta C T$ and A5 $\Delta$ CT showed only marginal binding to non-NAbs, b6, 412d and F105, 39F (Fig. 6c, e), respectively. Due to the lack of presence of epitopes for certain neutralizing antibodies in different Envs, we used a combination of different antibodies in Fig. 6a, c, e. In order to directly compare the effect of CT deletion of JRFL, JRCSF and A5 on its binding to bNAbs and non-NAbs and also to investigate whether the CD4i and immunodominant V3 epitopes are exposed in $\triangle \mathrm{CT}$ versions of these Envs, we studied their binding to the bNAb, PGT145 and non-NAbs, 17b and 19b (Fig. 6b, d, f). For this purpose, we used the JRFL mutant JRFLE168K as JRFL by itself does not bind PGT145. As shown in Fig. 6d, f, JRFLE168K and A5 and their $\triangle \mathrm{CT}$ counterparts bind to PGT145 efficiently but weakly to $17 \mathrm{~b}$ and 19b. However, JRCSF $\triangle$ CT binds more efficiently to the
non-NAbs, 17b and 19b as compared to PGT145 (Fig. 6b) suggesting that the immunodominant epitopes, CD4i and V3 are exposed in JRCSF $\Delta$ CT. Thus, the homologous Envs, JRFL and JRCSF, isolated from the same patient, showed opposite antigenic phenotype upon deletion of the C-terminus, indicating that the CT of Envs may regulate conformation and antigenicity differently. Results from all these studies suggest that the $\mathrm{CT}$ of individual efficiently cleaved, functional Envs has varied effects on their expression, conformation and antigenicity.

\section{JRFL $\Delta C T$, JRCSF $\Delta C T$ and A5 $\Delta C T$ are efficiently cleaved}

We next investigated whether the observed alteration in conformation/antigenicity of JRCSF $\Delta C T$ was due to inefficient cleavage of the $\triangle \mathrm{CT}$ version. The trimer selective, cleavage specific bNAbs, PGT151 and PGT145 show significant reduction in binding to uncleaved forms (cleavage site is mutated from REKR to SEKS) of the efficiently cleaved Envs, JRFL, JRCSF, 4-2.J41 and A5 [8, 20, 21]. Thus, efficient binding to these bNAbs is a good measure of cleavage efficiency. The Envs JRFLE168K, JRCSF, A5 and their $\triangle \mathrm{CT}$ variants bind efficiently to bNAb, PGT145 (Fig. 6b, d, f) suggesting that C-terminal truncation does not affect cleavage efficiency. We further verified that the $\Delta \mathrm{CT}$ forms of these Envs are efficiently cleaved by immunoprecipitation of plasma membrane (PM) fractions of transfected cells using cleavage independent bNAbs, VRC01 and PG9 (Additional file 3: Fig. S2). We used JRFL as a control as it shows only gp120 band from precipitates of biotinylated cell surface fractions of transfected cells and is therefore efficiently cleaved [8]. Previously using uncleaved (gp160 only band) and partially cleaved (both gp120 and gp160 bands) Envs as controls, we have shown that VRC01-immunoprecipitates of PM fraction of JRFL transfected cells gives only gp120 band in western blot $[19,21]$. Western blot analysis of the immunoprecipitates of JRFL with VRC01 used as control, and JRFL $\Delta$ CT, JRCSF $\Delta$ CT, 4-2.J41 $\Delta$ CT and A5 $\Delta$ CT with either VRC01 or PG9 shows gp120 band, with minor size variations possibly due to differential glycosylation (Additional file 3: Fig. S2). Taken together our studies strongly suggest that C-terminal deletion of efficiently 


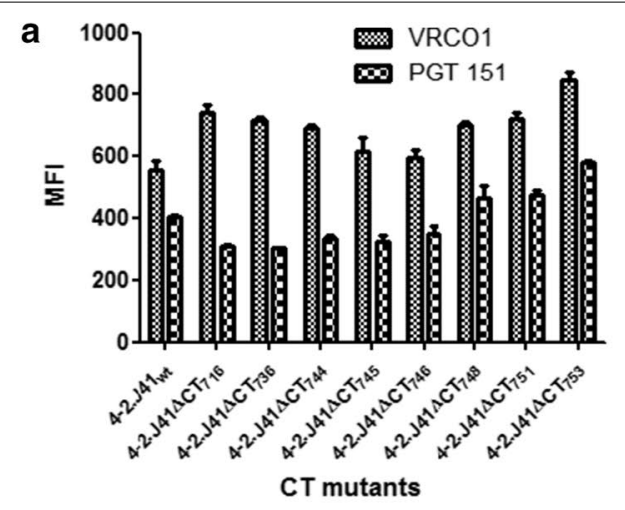

b
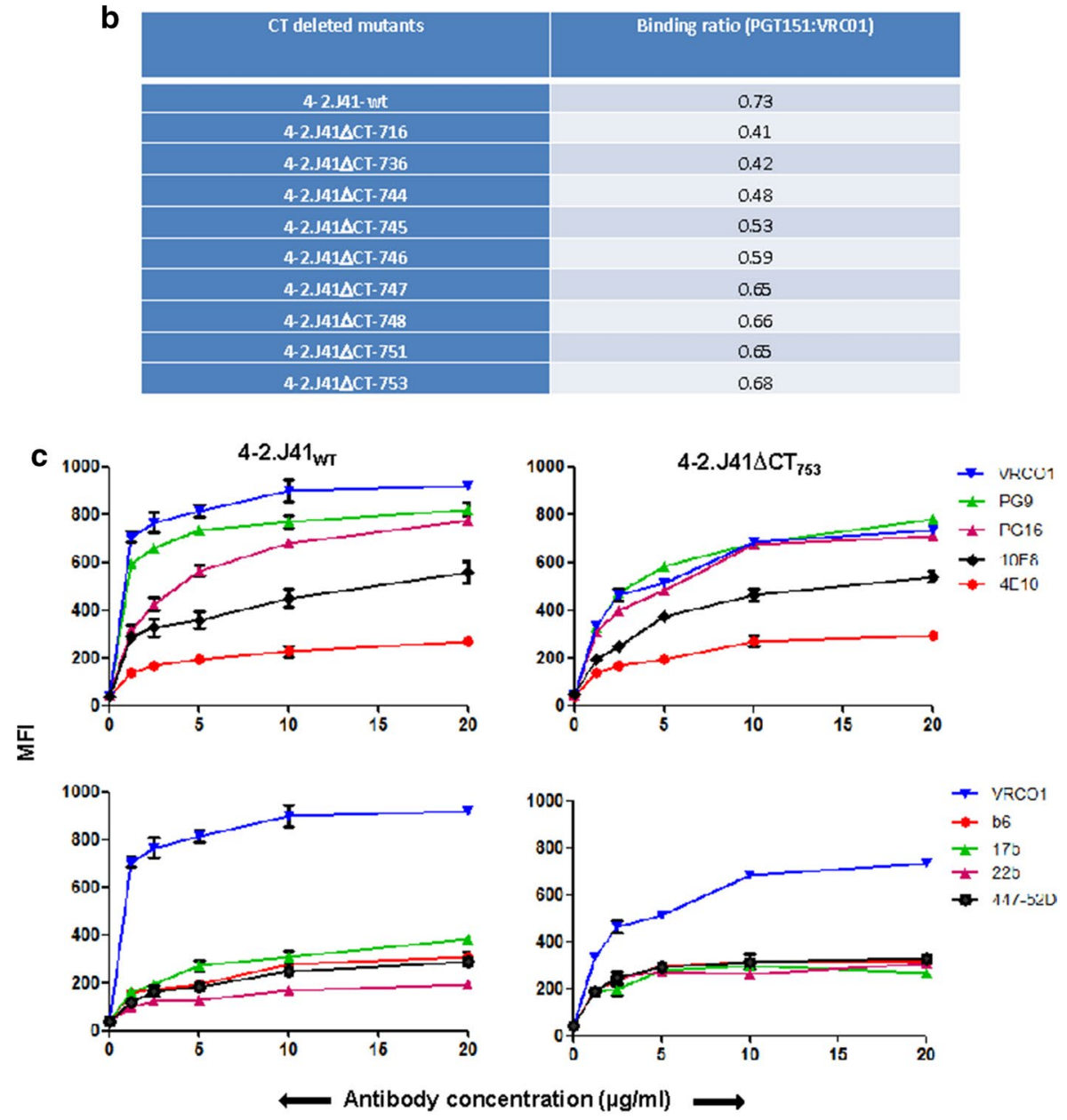

d
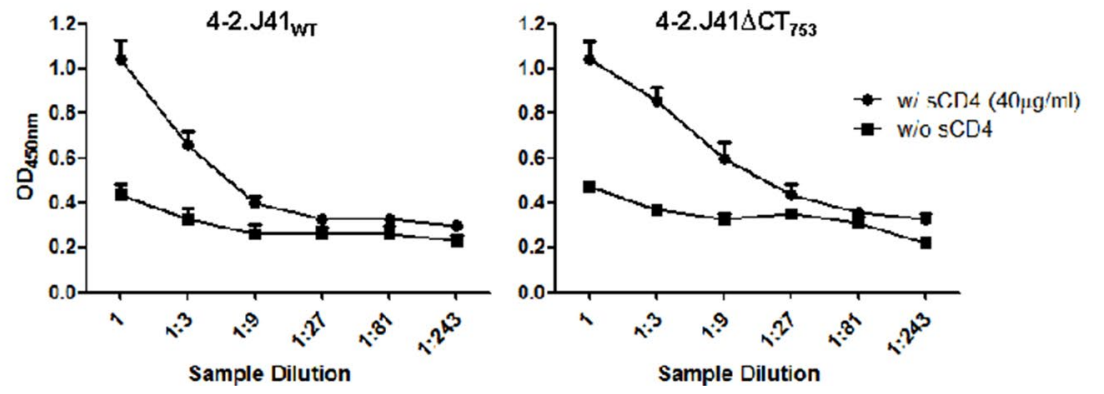

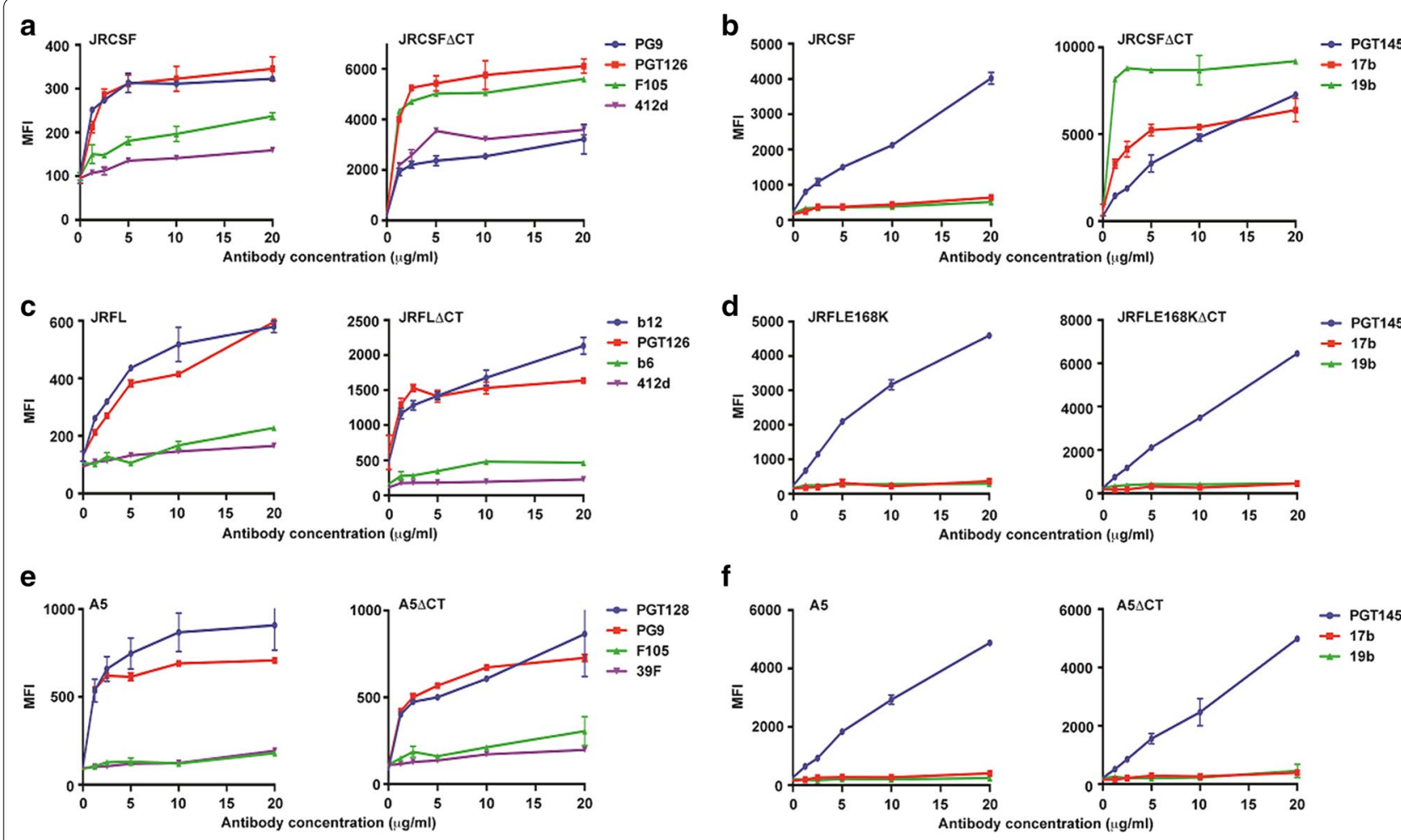

f
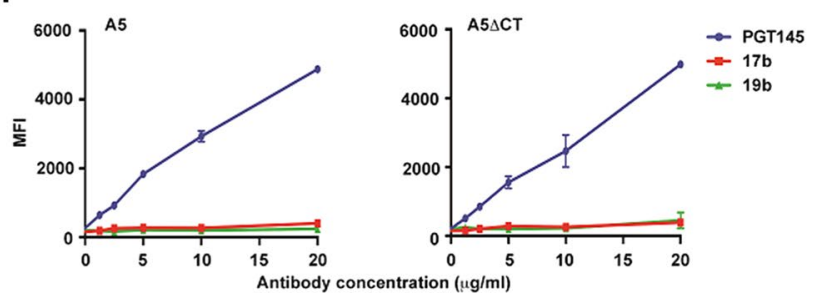

Fig. 6 CT-deleted JRCSF but not JRFL and A5, have altered cell surface antigenicity/conformation. a, c, e FACS based cell surface staining assays of JRCSF, JRCSF $\triangle C T$ with bNAbs, PGT126, PG9 and non-NAbs, F105, 412d; JRFL, JRFL $\triangle C T$ with bNAbs, b12, PGT126 and non-NAbs, b6 and 412 ; A5, A5 $\triangle$ CT with bNAbs, PGT128 and PG9 and non-NAbs, F105, 39F over a range of antibody concentrations. b, d, f FACS based cell surface staining assays of JRFLE168K, JRFLE168K $\triangle C T$, A5, A5 $\triangle C T$, JRCSF, JRCSF $\triangle C T$ with trimer-selective, cleavage-specific bNAb, PGT145 and non-NAbs, 17b (CD4i) and $19 \mathrm{~b}$ (V3) over a range of antibody concentrations

cleaved Envs JRFL, JRCSF and A5 do not affect their cleavage efficiency.

\section{Conserved hydrophilic domain in CT of JRCSF $\Delta \mathrm{CT}_{759}$ restores wild type conformation/antigenicity}

Next, we tested whether the region corresponding to the $\mathrm{CT}$ of 4-2.J41 $\Delta \mathrm{CT}_{753}$, containing the conserved hydrophilic domain, in JRCSF was able to restore wild type antigenicity and conformation. As 4-2.J41 $\Delta \mathrm{CT}_{759}$ showed the best result in terms of restoring native conformation and antigenicity we tested JRCSF $\Delta \mathrm{CT}_{759}$ for its ability to bind the bNAbs PGT121 (JRCSF does not bind efficiently to VRC01) and 2G12 (data not shown) and non-NAb 19b and compared it with JRCSF wild type (Fig. 7a). JRCSF $F_{W T}$ binds to PGT121 and 2G12 efficiently but weakly to $19 \mathrm{~b}$, and JRCSF $\Delta \mathrm{CT}_{759}$ was able to restore wild type properties as it binds efficiently to PGT121 and 2G12 but marginally to $19 \mathrm{~b}$ (Fig. 7a). Similarly JRCSF $\Delta \mathrm{CT}_{759}$ showed efficient binding to the trimer-selective, cleavage-specific bNAb PGT145 and weaker binding to the non-NAbs F105 and 17b (Fig. 7b) suggesting restoration of wild type properties. Taken together these studies suggest that the conserved hydrophilic domain containing region in
JRCSF $\Delta \mathrm{CT}_{759}$ restores wild type conformation and antigenicity similar to $4-2 . J 41 \Delta C \mathrm{~T}_{753}$.

\section{Determination of infectivity and antigenicity of cytoplasmic deleted mutant viruses}

To determine the effect of $\mathrm{CT}$ deletion on infectivity and neutralization sensitivity of 4-2.J41 viruses, we produced different 4-2.J41 CT-deleted Env pseudotyped viruses by transfection of $293 \mathrm{~T}$ cells with pSG3 $\Delta$ env vector backbone and Env plasmids [40]. Infectivity of the pseudotyped viruses was determined using TZM-bl reporter cells [52] by infecting with supernatants containing equal amounts of viral particles as measured by p24 ELISA (normalized to p24) and tested for luciferase activity after $48 \mathrm{~h}$. We consistently found that all CT-deleted mutant Env pseudotyped viruses show high luciferase titers (Fig. 8a, $P>0.05$ ) similar to that of wild type virus. Our results suggest that all the CT mutants are capable of viral entry and replication in TZM-bl cells. We further assessed the neutralization sensitivity of CT-deleted mutants to various monoclonal antibodies as described in Materials and Methods and determined the $\mathrm{IC}_{50}$ values $(\mu \mathrm{g} / \mathrm{ml})$. Among the various neutralizing mAbs we 

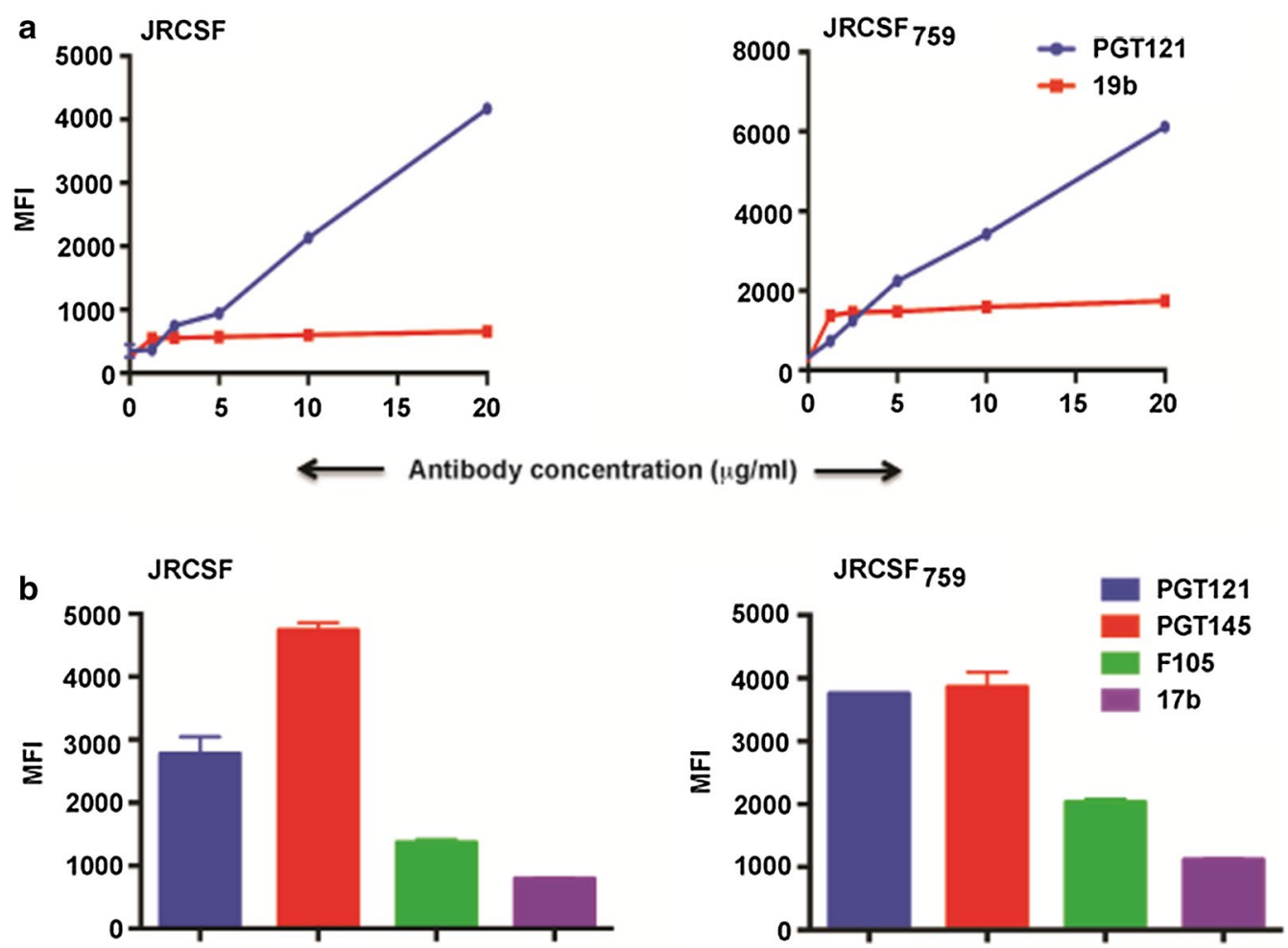

Fig. 7 Restoration of conformation/antigenicity in JRCSF $\triangle C T_{759}$ Env. a FACS based cell surface staining assays of JRCSF and JRCSF $\triangle C T_{759}$ with bNAb, PGT121 and non-NAb, 19b over a range of antibody concentration. b FACS based cell surface staining assays of JRCSF and JRCSF $\triangle C T_{759}$ with bNAbs, PGT121, PGT145 and non-NAbs, F105 and 17b at $20 \mu \mathrm{g} / \mathrm{ml}$ antibody concentration

tested, all the CT-deleted mutants were highly sensitive to VRCO1 (anti-CDbs) antibody with $\mathrm{IC}_{50}$ values $<2$ (Fig. 8b). In case of mAbs PG9 (anti-V2/V3) and PGT151 (cleavage and trimer specific), although the $\mathrm{IC}_{50}$ 's are $<4$, the CT mutant viruses show a 5-10 fold decrease in neutralization sensitivity as compared to wild type virus (Fig. 8b). Moreover, mAbs PGT121 and 10E8 affected the CT deleted mutants similarly (Fig. 8b, c), whereas the CT mutants showed more resistance to PGT145 (cleavage, quaternary specific) and 4E10 (MPER directed) $\mathrm{mAb}$ (Fig. 8b, c). The CT deleted mutant viruses showed neutralization resistance to non-NAbs (F105, 17b) with $\mathrm{IC}_{50}$ values $>30$ when antibody was incubated with the viruses at $37{ }^{\circ} \mathrm{C}$ followed by TZM-bl assay. However, when incubation was carried out at $4{ }^{\circ} \mathrm{C}$, the $\mathrm{CT}$ deleted mutant viruses were less resistant to non-NAbs (Fig. 8c, $P<0.0001)$. Our results suggest that at the virus level deletion of the CT of 4-2.J41 had no effect on infectivity and modest effects on neutralization sensitivity to bNAbs and non-NAbs. The conserved hydrophilic domain did not appear to restore the modest effects on neutralization sensitivity in 4-2.J41 $\Delta C \mathrm{~T}_{711}$ Env to wild type levels. With JRCSF $\triangle$ CT Env pseudotyped viruses, the neutralization sensitivity $\left(\mathrm{IC}_{50}\right.$ values) with the bNAbs VRC01,
PG9, PGT121, PGT151 and PGT145 was very similar to the wild type protein (Additional file 4: Fig. S3). Similar to wild type viruses, JRCSF $\triangle$ CT Env pseudotyped viruses were resistant to neutralization by the non-NAbs, F105 and 17b (Additional file 4: Fig S3). About a 5 fold higher sensitivity was observed for neutralization by $10 \mathrm{E} 8$ (MPER-directed bNAb) for JRCSF $\triangle C T$ Env pseudoytped virus as compared to JRCSF Env pseudotyped virus (Additional file 4: Fig S3). Thus, these studies suggest that sensitivity of JRCSF $\triangle$ CT pseudotyped viruses to various bNAbs and non-NAbs is not significantly different from that of virus pseudotyped with full length JRCSF Env.

\section{Discussion}

Several functions of Env are regulated by its C-terminal tail (CT) [30, 41, 42]. The unusually long conserved CT in HIV-1 Env mediates native conformation, and various Env functions, including trafficking, proper surface expression, membrane fusion, virion replication and budding [25, 26, 34, 43-45]. Among these functions, the effect of CT on expression, conformation and antigenicity are critical for developing Env-based immunogens. Studies on HIV-1 and SIV Envs have demonstrated that truncation of the $\mathrm{CT}$ alters the conformation of the 


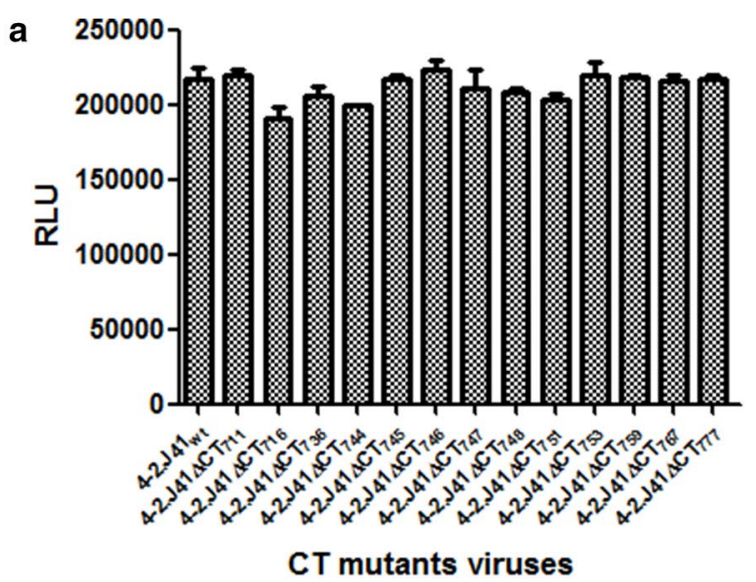

b

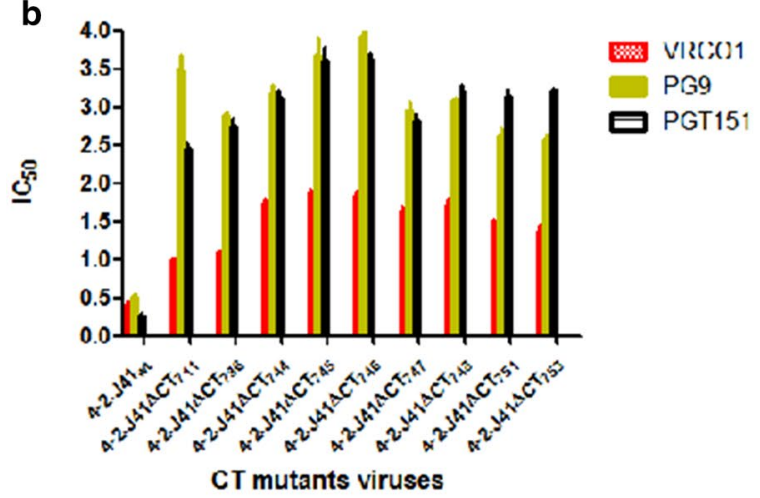

C

\begin{tabular}{|c|c|c|}
\hline \multirow[t]{2}{*}{ Viruses } & \multicolumn{2}{|c|}{$\mathrm{IC}_{50}(\mu \mathrm{g} / \mathrm{ml})$} \\
\hline & PGT145 & PGT121 \\
\hline 4-2.J41 & 7.12 & $<0.5$ \\
\hline 4-2.J41ACT 711 & $>10$ & $<0.5$ \\
\hline 4-2.J41 $\Delta \mathrm{CT}_{736}$ & $>10$ & $<0.5$ \\
\hline 4-2.J41 $\Delta \mathrm{CT}_{744}$ & $>10$ & $<0.5$ \\
\hline 4-2.J41 $\Delta \mathrm{CT}_{745}$ & $>10$ & $<0.5$ \\
\hline 4-2.J41 $\Delta \mathrm{CT}_{746}$ & $>10$ & $<0.5$ \\
\hline 4-2.J41 $\mathrm{CCT}_{747}$ & $>10$ & $<0.5$ \\
\hline 4-2.J41 $\Delta \mathrm{CT}_{748}$ & $>10$ & $<0.5$ \\
\hline 4-2.J41 $\Delta \mathrm{CT}_{751}$ & $>10$ & $<0.5$ \\
\hline 4-2.J41ACT 753 & $>10$ & $<0.5$ \\
\hline
\end{tabular}

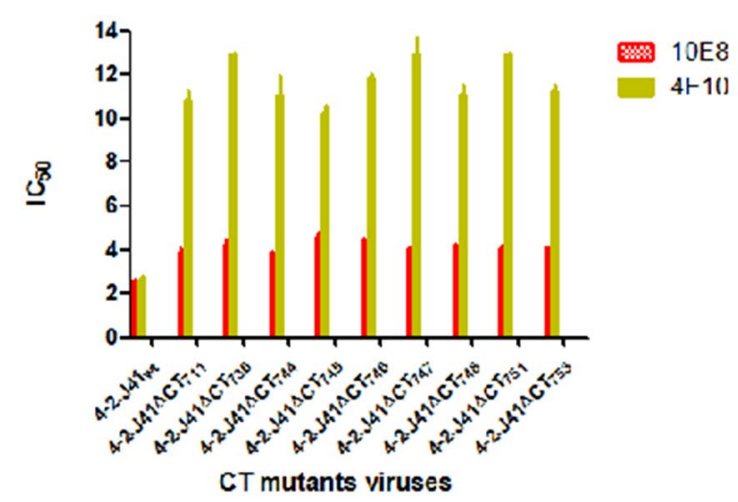

\begin{tabular}{|c|c|c|c|c|}
\hline \multirow[t]{2}{*}{ Viruses } & \multicolumn{4}{|c|}{$\mathrm{IC}_{50}(\mu \mathrm{g} / \mathrm{ml})$} \\
\hline & $\begin{array}{c}\mathrm{F} 105 \text { at } \\
37^{\circ} \mathrm{C}\end{array}$ & $\begin{array}{c}\mathrm{F} 105 \text { at } \\
4^{0} \mathrm{C}\end{array}$ & $\begin{array}{c}17 \mathrm{~b} \text { at } \\
37^{\circ} \mathrm{C}\end{array}$ & $\begin{array}{c}17 \mathrm{~b} \text { at } \\
4^{0} \mathrm{C}\end{array}$ \\
\hline 4-2.J41 & $>30$ & $>30$ & $>30$ & $>30$ \\
\hline 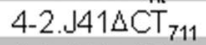 & $>30$ & 12 & $>30$ & 15 \\
\hline $4-2 . J 41 \Delta \mathrm{CT}_{736}$ & $>30$ & 18.56 & $>30$ & 13.05 \\
\hline $4-2 . J 41 \Delta C T_{744}$ & $>30$ & 14.5 & $>30$ & 12. \\
\hline 4-2.J41 $\Delta \mathrm{CT}_{745}$ & $>30$ & 12.39 & $>30$ & 14.5 \\
\hline 4-2.J41ACT 746 & $>30$ & 10.78 & $>30$ & 13.11 \\
\hline 4-2.J41 $\mathrm{CCT}_{747}$ & $>30$ & 9.45 & $>30$ & 12.56 \\
\hline 4-2.J41 $\Delta \mathrm{CT}_{748}$ & $>30$ & 11.9 & $>30$ & 13.78 \\
\hline 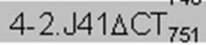 & $>30$ & 13.02 & $>30$ & 16.71 \\
\hline 4-2.J41 $\Delta \mathrm{CT}_{753}$ & $>30$ & 14. & $>30$ & 14.89 \\
\hline
\end{tabular}

Fig. 8 Infectivity and neutralization assays of 4-2.J41 mutant Env pseudotyped viruses. a Infectivity of 4-2.J41 Env wild type and deletion mutant pseudoviruses using TZM-bl reporter based cell assay. $\mathbf{b} \mid C_{50}$ values of 4-2.J41 wild type and deletion mutant Env pseudotyped viruses with the cleavage non-specific bNAb VRC01, glycan-dependent and conformational bNAb, PG9, trimer-selective and cleavage-specific bNAb, PGT151, MPERdirected bNAbs, $10 E 8$ and 4E10. $\mathbf{c} \mathrm{C}_{50}$ values of 4-2.J41 wild type and deletion mutant Env pseudotyped viruses with bNAbs, PGT121 and PGT145 (left panel) and non-NAbs F105 and $17 \mathrm{~b}$ at 37 and $4^{\circ} \mathrm{C}$ (right panel) 
ectodomain but the exact mechanism is poorly understood [29-31]. Previously, it has been suggested, that for partially cleaved Envs, removal of the C-terminus results in increase in expression and the exposure of epitopes for non-neutralizing antibodies [32]. In addition, CT truncation have been reported to affect binding to conformational mAbs, CD4bs-targeting and CD4i-directed antibodies in comparison to full-length protein [30] although the cleavage status of these Envs has not been reported.

Efficient cleavage of gp160 Envs into their constituent subunits gp120-gp41 is a pre-requisite for selective exposure of bNAb-binding epitopes and is thus the closest mimic of native, functional Envs - a property desirable for immunogen development. The CT of Envs is often deleted in order to enhance the expression and also to stabilize the soluble form of native-like trimeric Env immunogens $[11,14,28]$. The identification of the four efficiently cleaved Envs (JRFL, JRCSF, 4-2.J41 and A5) from clades A, B and C $[8,20,21]$ allowed us to analyze the effect of deleting the $\mathrm{C}$-terminus on the expression, conformation and antigenicity of these cross clade Envs, parameters important for designing immunogens for DNA, viral vector, virus-like particle as well as soluble protein based vaccination. We find that CT-deleted mutants of efficiently cleaved Envs display phenotypic diversity. While the expression of JRFL, JRCSF and 4-2. $\mathrm{J} 41$ is increased, the expression of A5 does not significantly change even though it retains the two major endocytosis signals in its CT. Conformation and antigenicity of C-terminus deleted JRFL and A5 does not change but in the case of JRCSF and 4-2.J41, non-neutralizing epitopes get exposed upon deletion of CT. We also find that the homologues JRFL and JRCSF behave differently when their CT is deleted.

The precursor gp160 polypeptide of HIV-1 Env is synthesized in ER and transported to the Golgi/trans-Golgi network for post translational modification and cleavage to attain native conformation and thus generate efficiently cleaved, functional Envs [44, 46]. It is possible that JRCSF $\Delta$ CT and 4-2.J41 $\Delta$ CT Env is inefficiently cleaved, which exposes epitopes for non-NAbs. However, our data demonstrates that there is no significant reduction in cleavage of $\Delta \mathrm{CT}$ forms of efficiently cleaved Envs. This led us to investigate the role of different domains and regions in the CT of 4-2.J41 and JRCSF that may be required for maintaining native-like ectodomain conformation/antigenicity of the wild type proteins. The role of different conserved domains in the CT of Env in its functions is not fully understood. In this study, we have used deletion constructs and cell surface staining to map the conserved domain(s) in the CT of 4-2.J41 that can restore wild type antigenicity/conformation. The CT of 4-2.J41 Env contains three conserved domains called lentivirus lytic peptides (LLP2, LLP3, and LLP1) that show high degree of conservation among CTs of different HIV-1 strains and also among CTs of other lentivirus Envs such as HIV-2 and SIV [24, 34, 47]. Deletion of LLP domains in CT of 4-2.J41 did not alter the integrity of its ET. We evaluated the role of another highly conserved hydrophilic domain $[22,48]$ in restoring the integrity of 4-2.J41 Env and JRCSF Env ET [24, 35, 42]. Using in silico analysis, we confirmed the high hydrophilicity of this conserved region in the CT of 4-2.J41 Env between amino acids 724-745 and we termed it as conserved hydrophilic domain (CHD). Restoration of the CHD-containing region in 4-2.J41 $\Delta \mathrm{CT}_{753}$ restored the native, wild-type conformation and antigenicity of ET. 4-2.J41 $\Delta \mathrm{CT}_{753}$ Env containing the CHD was sufficient to restore conformation of the ectodomain as evaluated by efficient binding to VI/V2 quaternary epitope targeted mAbs, PG9 and PG16, MPER-directed mAbs, 4E10 and 10E8 and marginal binding to several non-NAbs. Similarly, restoration of the conserved hydrophilic region at the C-terminus in JRCSF $\Delta \mathrm{CT}_{759}$ restored the ET conformation/antigenicity to wild type protein. To the best of our knowledge a role for the conserved hydrophilic domain in Env ectodomain conformation and antigenicity has not been previously demonstrated. Taken together these studies for the first time demonstrates a role for the conserved hydrophilic domain in the C-terminal tail of a subset of functional Envs in maintaining their ectodomain conformation/antigenicity. Furthermore, 4-2.J41 CT-deleted Env pseudotyped virus particles showed only modest neutralization sensitivity variation to different bNAbs and modest temperature dependent sensitivity to non-NAbs. These effects were not restored to wild type levels by restoring the conserved hydrophilic domain in 4-2.J41 $\Delta \mathrm{CT}_{753}$ Env pseudotyped viruses. The neutralization sensitivity of JRCSF and JRCSF $\triangle$ CT pseudotyped viruses to different bNAbs and non-NAbs were found to be similar with an exception for 10E8. Thus, these results suggest that effect of the conserved hydrophilic domain in regulating the antigenicity and conformation of 4-2.J41 and JRCSF Envs is limited to the cell surface expressed protein. Based on these results we hypothesize that deletion of the CT in 4-2.J41 and JRCSF Envs affects their native structure which leads to the generation of a heterogeneous population of Envs like native, near-native and 
non-native molecules within the cell but in the presence of one or more viral protein(s) only the native or nearnative $\Delta C T$ Envs are incorporated into viral particles. Viral and cellular factors play important roles in HIV-1 particle assembly [44]. Gag plays a critical role in this process and MA (matrix) directs incorporation of Env into virions during assembly [44]. Two of the proposed Env incorporation into virion models posits that Env is taken up in assembling viruses through direct association or a host protein-mediated interaction with Gag [44]. Thus, there may be a mechanism during the assembly of viral particles to preferentially incorporate functional Envs. Some degree of incorporation of non-native forms can be seen in these cell lines e.g. incorporation of small amounts of uncleaved Envs in virus-like particles [28]. The conserved hydrophilic domain, also called Kennedy epitope, has been proposed to be intracytoplasmic in the traditional model while in the alternate model this region is suggested to be extracellular [48]. However, the NMR structure of the region containing the conserved hydrophilic domain from the pNL4-3 isolate shows that it is unstructured and not associated with membrane [49] although it is still possible that the characteristics of this domain is strain-specific. Furthermore, in the context of the virus, the $\mathrm{CHD}$ alone may not be sufficient to restore wild type properties as it is evident by the inability of 4-2.J41 $\Delta \mathrm{CT}_{753}$ Env pseudotyped viruses to maintain the neutralization sensitivity like the 4-2.J41 Env pseudotyped virus. Therefore, how and why this domain determines ET integrity in only some but not all efficiently cleaved Envs need to be investigated. Finally, including the conserved hydrophilic domain in Env-based soluble immunogen design may be necessary for Envs whose CT regulate their ET antigenicity and conformation on the plasma membrane.

It is to be noted here that we have looked at the effect of CT deletion on efficiently cleaved Envs in the permissive cell line $293 \mathrm{~T}$ as this cell line and its derivatives are frequently used to produce Env-based immunogens. Furthermore, the $\Delta \mathrm{CT}$ Envs are efficiently incorporated into viral particles in this cell line and thus making it easier to compare the neutralization sensitivity against a number of antibodies to virus pseudotyped with full length Env. Furthermore, most of the assays used for this study are well established in 293T cell line. Besides from a vaccine immunogen development point of view, study in 293T cells or their derivatives may be relevant. However, $\mathrm{T}$ cells, peripheral blood mononuclear cells (PBMCs) and monocyte-derived macrophages are the natural targets for HIV-1 infection and the CT of Env is required for spreading productive infection in these cell types [50]. So in order to better understand the biological role of the CT and the conserved hydrophilic domain of the efficiently cleaved Envs, used in this study, in determining Env ET antigenicity/conformation, it will be necessary to extend this study to these cell types in future.

\section{Conclusion}

The long C-terminal tail of Env contains several domains and plays an important role in multiple functions of the protein including in maintaining its ectodomain antigenicity and conformation. Using four efficiently cleaved, functional Envs we show that deletion of the CT has variable effect on the ectodomain antigenicity and conformation with a subset represented by 4-2.J41 and JRCSF Envs being affected while the Envs JRFL and A5 remain unaffected. Using deletion analysis we find that a region in the CT containing a conserved hydrophilic domain restores native ectodomain antigenicity and conformation in 4-2. J41 and JRCSF. This effect is restricted to the plasma membrane bound Env and is not seen in Env on the viral membrane. This study demonstrates the importance of the conserved hydrophilic domain in maintaining the integrity of the ectodomain on the cell surface and will help further guide to design native-like functional Envbased immunogens.

\section{Methods \\ Cell lines and antibodies}

293T cells were purchased from the American Type Culture Collection (ATCC). TZM-bl cells were obtained from NIH AIDS Reagent Program. The cells were maintained in Dulbecco's modified Eagle's medium (DMEM) (Invitrogen, USA) supplemented with $10 \%$ fetal calf serum (HiMedia, USA), $20 \mathrm{mM}$ L-glutamine, $100 \mathrm{U} /$ $\mathrm{ml}$ penicillin, and $100 \mu \mathrm{g} / \mathrm{ml}$ streptomycin. The broadly neutralizing antibodies (VRC01, PGT121/128, PGT145, PGT151, PG9, PG16, 10E8 and 4E10) and non-neutralizing antibodies (F105, b6, 39F, 447-52D, 22b and 17b) were obtained from the IAVI Neutralizing Antibody Center (NAC) at TSRI, La Jolla, California. The antigp120 (clades A, B, C) rabbit polyclonal antibodies were purchased from ABLinc, USA.

\section{Plasmids and mutagenesis}

The full-length Env 4-2.J41 (gp160) clones were obtained from National AIDS Research Institute, Pune, India and have been previously described [51]. To generate CT deleted constructs, stop codons were introduced into the cytoplasmic tail at amino acid (aa) position 712 (4-2. $\left.\mathrm{J} 41 \Delta \mathrm{CT}_{711}\right), 717$ (4-2.J41 $\left.\Delta \mathrm{CT}_{716}\right), 737$ (4-2.J41 $\left.\Delta \mathrm{CT}_{736}\right)$, 745 (4-2.J41 $\left.\Delta \mathrm{CT}_{744}\right), 746$ (4-2.J41 $\left.\Delta \mathrm{CT}_{745}\right), 747 \quad$ (4-2. J41 $\left.\Delta \mathrm{CT}_{746}\right), 749$ (4-2.J41 $\left.\Delta \mathrm{CT}_{748}\right), 752\left(4-2 . J 41 \Delta \mathrm{CT}_{751}\right)$, $754 \quad\left(4-2 . J 41 \Delta \mathrm{CT}_{753}\right), 760$ (4-2.J41 $\left.\Delta \mathrm{CT}_{759}\right), 768$ (4-2. $\left.\mathrm{J}_{41} \Delta \mathrm{CT}_{767}\right), 778$ (4-2.J41 $\left.\Delta \mathrm{CT}_{777}\right)$ in pSVIII-env plasmid backbone by PCR amplification using Phusion 
polymerase following manufacturer's protocol. The reaction mixtures were digested with $D p n 1$, transformed into competent cells and plated onto LB-ampicillin plates. The mutants were confirmed by sequencing of the plasmid DNA. JRCSF $\triangle$ CT and JRCSF $\Delta \mathrm{CT}_{759}$ were generated by introducing stop codons as described above.

\section{Cell surface assay using FACS-based method}

All flow cytometry-based cell surface assays were carried out as described previously [20]. 293T cells were transfected, harvested and then washed three times with FACS buffer I (DMEM $+5 \%$ HI-FBS). Cells were stained with increasing concentration of different bNAbs and nonNAbs in 96 well U-bottomed plate wells for $1 \mathrm{~h}$ at room temperature (RT). The cells were then washed with FACS buffer I, followed by incubation with PE-conjugated goat anti-human secondary antibody (1:200 dilutions, Jackson ImmunoResearch) for $1 \mathrm{~h}$. at RT. Next, the cells were washed with FACS buffer II (PBS + 5\% HI-FBS) and fixed with $0.5 \%$ paraformaldehyde. Finally, the stained and fixed cells were analyzed in a FACS Canto analyzer (BD Biosciences) and MFI determined by using FlowJo software (version 10.0.6, Tree Star Inc.).

\section{Analysis of sCD4-induced gp 120 shedding}

Soluble CD4 induced (sCD4-induced) shedding of gp120 was assayed as previously described [5, 20]. Briefly, 293T cells transfected with different plasmids harboring wild type or mutant Envs were harvested and washed with FACS buffer and then incubated with or without $50 \mu \mathrm{g} /$ $\mathrm{ml} \mathrm{sCD} 4$ (NIH AIDS Reagent) for $1 \mathrm{~h}$ at $4{ }^{\circ} \mathrm{C}$ with intermittent mixing of samples. Cells were centrifuged and the supernatant subjected to ELISA as described previously [20]. Supernatants were also subjected to western blot analysis anti-clade C Env gp120 polyclonal antibody.

Western blot analysis and cell surface protein biotinylation 293T cells were transiently transfected with Env constructs and $48 \mathrm{~h}$ post transfection whole cell extracts were isolated using lysis buffer, the lysed proteins were denatured, reduced, separated by $10 \%$ SDS-PAGE, and analyzed by Western blotting using a 1:200 dilution of anti-gp120 (clade C) rabbit polyclonal antibody.

For cell surface biotinylation, the methods were performed as described previously with few modifications [20]. Briefly, 293T cells were co-transfected with plasmids expressing with CT deleted or wild type Env and Tat proteins as described above. $36-48 \mathrm{~h}$ post transfection the cells were harvested and washed with PBS $(\mathrm{pH}$ 8 ). The cells were labeled with freshly prepared 2-5 mM biotin (EZ-link Sulfo-NHS-LC-Biotin, Thermo Scientific, and Cat. No. 21335) in PBS (pH 8) for $30 \mathrm{~min}$ at $4{ }^{\circ} \mathrm{C}$, washed with PBS twice, incubated with $50 \mathrm{mM}$ glycine in PBS (pH 7.5) for $30 \mathrm{~min}$ at $4{ }^{\circ} \mathrm{C}$ with rotation and then washed twice with PBS. Cell pellets were resuspended in RIPA buffer (50 mM Tris-HCI pH 7.4, $25 \mathrm{ml}$ of $1 \mathrm{M}$. $1 \%$ NP-40 $5 \mathrm{ml}$. 0.5\% Na-deoxycholate $2.5 \mathrm{~g}, 0.1 \%$ SDS, $150 \mathrm{mM} \mathrm{NaCl}, 2 \mathrm{mM}$ EDTA containing protease inhibitors) and incubated on ice for $30 \mathrm{~min}$. The cell extract was centrifuged at $13000 \mathrm{rpm}$ for $30 \mathrm{~min}$ at $4{ }^{\circ} \mathrm{C}$ and the supernatant was subjected to precipitation with $100 \mu \mathrm{l}$ slurry of high capacity neutravidin agarose (Thermo Scientific cat. no. 29204) (pre-washed with PBS) for $2 \mathrm{~h}$ at RT with rotation. Finally beads were washed three times with PBS $+1 \%$ Triton-X and washed beads were subjected to western blot analysis using rabbit anti-clade $\mathrm{C}$ antibodies as probes.

\section{p24 ELISA and HIV-1 infectivity assay (TZM-bl)}

Mutant pseudotyped viruses were produced from 293T cells by transient transfection with Fugene (Promega,USA) as previously described [52]. The p24 values were measured using HIV-1 p24 Antigen Capture Kit (abcam USA) following the manufacturer's instructions. Briefly, properly diluted virus samples were lysed and captured in a micro-ELISA plate at $37^{\circ} \mathrm{C}$ for $1 \mathrm{~h}$. The wells were then washed and the specifically captured p 24 antigen was incubated with human anti-p24 polyclonal antibodies conjugated with peroxidase at $37{ }^{\circ} \mathrm{C}$ for $1 \mathrm{~h}$. At the end of incubation, peroxidase substrate was added and the reaction was continued for $30 \mathrm{~min}$ at room temperature. The reaction was stopped by adding stop solution and OD read at $450 \mathrm{~nm}$.

For determination of HIV-1 infectivity, briefly, TZM-bl cells were seeded in 96-well plates at $10^{4}$ cells per well in $100 \mu \mathrm{l}$ complete DMEM and incubated for $24 \mathrm{~h}$ at $37^{\circ} \mathrm{C}$. Virus samples normalized to p24 value were added to cells in a total volume per well of $200 \mu \mathrm{L}$ DMEM. Cells were harvested $48 \mathrm{~h}$ post-infection, and HIV-1 LTRinduced luciferase activity in the cells was determined using the Luciferase Assay System (Promega). Results are reported in relative luminescence units (RLU) as measured on a luminometer (PerkinElmer).

\section{Calculations and statistics}

The Mean fluorescent intensity of a given experiment was calculated by finding average of duplicate wells and each experiment was repeated three times. Statistical analyses were performed using Prism, version 5.0, software (GraphPad Software). Error bars represent the standard errors of the mean (SEMs) of at least two independent 
experiments. Values were determined by Two-way ANOVA of pooled data.

\section{Additional files}

Additional file 1: Table S1a. Ratio of binding to non-NAbs versus VRC01. Table S1 b. Ratio of binding to bNAbs versus VRC01. Table S1c. Ratio of binding to PGT 151 versus VRC01.

Additional file 2: Fig. S1. Domains and endocytosis signals in JRFL, JRCSF, 4-2.J41 and A5. (a) Sequence comparisons of the C-terminal tails of 4-2.J41 (clade A), JRFL and JRCSF (clade B), A5 (clade A) and HxB2 showing that the endocytosis signals (highlighted in yellow) are largely conserved. Amino acid changes are marked in red. CHD (KE): Conserved Hydrophilic Region (Kennedy Epitope); LLP1, LLP2, LLP3 (Lentivirus Lytic Peptides $1,2,3)$. (b) Bright field image of $A 5$ and $A 5 \triangle C T$ transfected whole cells.

Additional file 3: Fig. S2. Cleavage properties of $\triangle C T$ mutants of JRFL, JRCSF, 4-2.J41 and A5. Western blot analysis of immunoprecipitates (with cleavage non-specific bNAbs VRC01 and PG9) of plasma membrane fractions of JRFL, JRFL $\triangle C T$, JRCSF $\triangle C T$, 4-2.J41 $\Delta C T$, A5 $\Delta C T$ transfected $293 T$ cells using rabbit anti-clades $\mathrm{A}, \mathrm{B}$ and $\mathrm{C}$ antibodies as probes.

Additional file 4: Fig. S3. Infectivity and neutralization assays of JRCSF and JRCSF $\triangle C T$ pseudoviruses. (a) Infectivity of JRCSF Env wild type and JRCSF $\triangle C T$ mutant pseudoviruses using TZM-bl reporter based cell assay. (b) $I_{50}$ values of JRCSF wild type and JRCSF $\triangle C T$ mutant pseudoviruses with the cleavage non-specific bNAb VRC01, glycan-dependent and conformational bNAb PG9, PGT121, trimer-selective and cleavage-specific bNAbs PGT151 and PGT145, MPER-directed bNAbs 10 E8 and non-NAbs F105 and 17b.

\section{Abbreviations}

HIV-1: human immunodeficiency virus type I; Env: envelope; bNAb: broadly neutralizing antibody; non-NAb: non-neutralizing antibody; FACS: fluorescence activated cell sorting; PM: plasma membrane; IP: immunoprecipitation; CD4i: CD4 induced; CT: C-terminal tail; ET: ectodomain.

\section{Authors' contributions}

SS and SD performed majority of the experiments, interpreted data, wrote the manuscript along with BKC. SB and HQ performed some FACS assays and interpreted FACS-based staining data, TS helped with experiments. NK, SG and MB prepared reagents. BKC designed, directed, interpreted all the experiments and wrote the manuscript along with SS and SD. All authors read and approved the final manuscript.

\section{Author details}

${ }^{1}$ THSTIIAVI HIV Vaccine Design Program, Translational Health Science and Technology Institute, NCR Biotech Science Cluster, 3rd Milestone, Faridabad-Gurgaon Expressway, P.O. Box \# 04, Faridabad, Haryana 1221001, India. ${ }^{2}$ IAVI Neutralizing Antibody Center, The Scripps Research Institute, La Jolla, CA, USA. ${ }^{3}$ Innovation \& Translation, ABL, Inc., 9800 Medical Center Drive, Building D, Rockville, MD 20850, USA.

\section{Acknowledgements}

We would like to thank NAC for providing some of the reagents for this study. We would like to thank Nigel Dimmock, University of Warwick Ronald C. Montelaro (University of Pittsburgh School of Medicine) for the SAR-1 antibody. We would also like to thank NIH AIDS REAGENT PROGRAM for 1577 antibody. We would like to thank Shreyasi Chatterjee for helping us in getting reagents and support.

\section{Competing interests}

The authors declare that they have no competing interests.

\section{Availability of data and materials}

Readily available through preservation of intellectual rights.
Consent for publication

Not applicable.

\section{Ethics approval and consent to participate}

Not applicable.

\section{Funding}

This work was supported primarily by IAVI intramural research, as well as by the Department of Biotechnology (DBT), Govt. of India intramural research program and its generous donors. IAVI's work is made possible by generous support from many donors including: the Bill \& Melinda Gates Foundation; the Ministry of Foreign Affairs of Denmark; Irish Aid; the Ministry of Finance of Japan; the Ministry of Foreign Affairs of the Netherlands; the Norwegian Agency for Development Cooperation (NORAD); the United Kingdom Department for International Development (DFID), and the United States Agency for International Development (USAID). The full list of IAVI donors is available at www.iavi.org. This study is made possible by the generous support of the Bill \& Melinda Gates Foundation Collaboration for AIDS Vaccine Discovery and the American people through USAID. The contents are the responsibility of the International AIDS Vaccine Initiative and do not necessarily reflect the views of USAID or the United States Government.

\section{Publisher's Note}

Springer Nature remains neutral with regard to jurisdictional claims in published maps and institutional affiliations.

Received: 7 April 2018 Accepted: 3 July 2018

Published online: 20 July 2018

\section{References}

1. Hallenberger S, Bosch V, Angliker H, Shaw E, Klenk HD, Garten W. Inhibition of furin-mediated cleavage activation of HIV-1 glycoprotein gp 160 . Nature. 1992;360(6402):358-61.

2. Poignard P, Saphire EO, Parren PW, Burton DR. gp120: Biologic aspects of structural features. Annu Rev Immunol. 2001;19:253-74.

3. Wyatt R, Sodroski J. The HIV-1 envelope glycoproteins: fusogens, antigens, and immunogens. Science. 1998;280(5371):1884-8.

4. Guenaga J, de Val N, Tran K, Feng Y, Satchwell K, Ward AB, Wyatt RT. Wellordered trimeric HIV-1 subtype B and C soluble spike mimetics generated by negative selection display native-like properties. PLoS Pathog. 2015;11(1):e1004570.

5. Chakrabarti BK, Pancera M, Phogat S, O'Dell S, McKee K, Guenaga J, Robinson J, Mascola J, Wyatt RT. HIV type 1 Env precursor cleavage state affects recognition by both neutralizing and nonneutralizing gp41 antibodies. AIDS Res Hum Retroviruses. 2011;27(8):877-87.

6. Ringe RP, Sanders RW, Yasmeen A, Kim HJ, Lee JH, Cupo A, Korzun J, Derking $R$, van Montfort T, Julien JP, et al. Cleavage strongly influences whether soluble HIV-1 envelope glycoprotein trimers adopt a native-like conformation. Proc Natl Acad Sci USA. 2013;110(45):18256-61.

7. Yasmeen $A$, Ringe R, Derking R, Cupo A, Julien JP, Burton DR, Ward AB, Wilson IA, Sanders RW, Moore JP, et al. Differential binding of neutralizing and non-neutralizing antibodies to native-like soluble HIV-1 Env trimers, uncleaved Env proteins, and monomeric subunits. Retrovirology. 2014;11:41.

8. Pancera M, Wyatt R. Selective recognition of oligomeric HIV-1 primary isolate envelope glycoproteins by potently neutralizing ligands requires efficient precursor cleavage. Virology. 2005;332(1):145-56.

9. Crooks ET, Moore PL, Franti M, Cayanan CS, Zhu P, Jiang P, de Vries RP, Wiley C, Zharkikh I, Schulke N, et al. A comparative immunogenicity study of HIV-1 virus-like particles bearing various forms of envelope proteins, particles bearing no envelope and soluble monomeric gp120. Virology. 2007;366(2):245-62

10. Zhu P, Liu J, Bess J Jr, Chertova E, Lifson JD, Grise H, Ofek GA, Taylor KA, Roux KH. Distribution and three-dimensional structure of AIDS virus envelope spikes. Nature. 2006;441(7095):847-52.

11. Sanders RW, Derking R, Cupo A, Julien JP, Yasmeen A, de Val N, Kim HJ, Blattner C, de la Pena AT, Korzun J, et al. A next-generation cleaved, 
soluble HIV-1 Env trimer, BG505 SOSIP.664 gp140, expresses multiple epitopes for broadly neutralizing but not non-neutralizing antibodies. PLoS Pathog. 2013;9(9):e1003618.

12. Sanders RW, van Gils MJ, Derking R, Sok D, Ketas TJ, Burger JA, Ozorowski G, Cupo A, Simonich C, Goo L, et al. HIV-1 VACCINES. HIV-1 neutralizing antibodies induced by native-like envelope trimers. Science. 2015;349(6244):aac4223.

13. Georgiev IS, Joyce MG, Yang Y, Sastry M, Zhang B, Baxa U, Chen RE, Druz A, Lees CR, Narpala S, et al. Single-chain soluble BG505. SOSIP gp140 trimers as structural and antigenic mimics of mature closed HIV-1 Env. J Virol. 2015:89(10):5318-29.

14. Sharma SK, de Val N, Bale S, Guenaga J, Tran K, Feng Y, Dubrovskaya V, Ward AB, Wyatt RT. Cleavage-independent HIV-1 Env trimers engineered as soluble native spike mimetics for vaccine design. Cell Rep. 2015;11(4):539-50.

15. Kong L, He L, de Val N, Vora N, Morris CD, Azadnia P, Sok D, Zhou B, Burton DR, Ward AB, et al. Uncleaved prefusion-optimized gp 140 trimers derived from analysis of HIV-1 envelope metastability. Nat Commun. 2016;7:12040

16. Vaine M, Wang S, Crooks ET, Jiang P, Montefiori DC, Binley J, Lu S. Improved induction of antibodies against key neutralizing epitopes by human immunodeficiency virus type 1 gp120 DNA prime-protein boost vaccination compared to gp120 protein-only vaccination. J Virol. 2008;82(15):7369-78.

17. Law M, Maruyama T, Lewis J, Giang E, Tarr AW, Stamataki Z, Gastaminza $\mathrm{P}$, Chisari FV, Jones IM, Fox RI, et al. Broadly neutralizing antibodies protect against hepatitis $C$ virus quasispecies challenge. Nat Med. 2008; 14(1):25-7.

18. Chakrabarti BK, Feng Y, Sharma SK, McKee K, Karlsson Hedestam GB, Labranche CC, Montefiori DC, Mascola JR, Wyatt RT. Robust neutralizing antibodies elicited by HIV-1 JRFL envelope glycoprotein trimers in nonhuman primates. J Virol. 2013;87(24):13239-51.

19. Das S, Boliar S, Samal S, Ahmed S, Shrivastava T, Shukla BN, Goswami S, Bansal M, Chakrabarti BK. Identification and characterization of a naturally occurring, efficiently cleaved, membrane-bound, clade A HIV-1 Env, suitable for immunogen design, with properties comparable to membranebound BG505. Virology. 2017;510:22-8.

20. Boliar S, Das S, Bansal M, Shukla BN, Patil S, Shrivastava T, Samal S, Goswami S, King CR, Bhattacharya J, et al. An efficiently cleaved HIV-1 clade C Env selectively binds to neutralizing antibodies. PLOS ONE. 2015;10(3):e0122443.

21. Das S, Boliar S, Mitra N, Samal S, Bansal M, Koff WC, Chakrabarti BK. Membrane bound modified form of clade B Env, JRCSF is suitable for immunogen design as it is efficiently cleaved and displays all the broadly neutralizing epitopes including V2 and C2 domain-dependent conformational epitopes. Retrovirology. 2016;13(1):81.

22. Postler TS, Desrosiers RC. The tale of the long tail: the cytoplasmic domain of HIV-1 gp41. JVirol. 2013;87(1):2-15.

23. Piller SC, Dubay JW, Derdeyn CA, Hunter E. Mutational analysis of conserved domains within the cytoplasmic tail of gp41 from human immunodeficiency virus type 1: effects on glycoprotein incorporation and infectivity. J Virol. 2000;74(24):11717-23.

24. Santos da Silva E, Mulinge M, Perez-Bercoff D. The frantic play of the concealed HIV envelope cytoplasmic tail. Retrovirology. 2013;10:54.

25. Breed MW, Jordan AP, Aye PP, Lichtveld CF, Midkiff CC, Schiro FR, Haggarty BS, Sugimoto C, Alvarez X, Sandler NG, et al. Loss of a tyrosine-dependent trafficking motif in the simian immunodeficiency virus envelope cytoplasmic tail spares mucosal CD4 cells but does not prevent disease progression. J Virol. 2013;87(3):1528-43.

26. Day JR, Munk C, Guatelli JC. The membrane-proximal tyrosine-based sorting signal of human immunodeficiency virus type $1 \mathrm{gp} 41$ is required for optimal viral infectivity. J Virol. 2004;78(3):1069-79.

27. Day JR, Van Damme N, Guatelli JC. The effect of the membrane-proxima tyrosine-based sorting signal of HIV-1 gp41 on viral infectivity depends on sequences within gp120. Virology. 2006;354(2):316-27.

28. Tong T, Crooks ET, Osawa K, Binley JM. HIV-1 virus-like particles bearing pure env trimers expose neutralizing epitopes but occlude nonneutralizing epitopes. J Virol. 2012:86(7):3574-87.

29. Spies CP, Ritter GD Jr, Mulligan MJ, Compans RW. Truncation of the cytoplasmic domain of the simian immunodeficiency virus envelope glycoprotein alters the conformation of the external domain. J Virol. 1994;68(2):585-91.

30. Edwards TG, Wyss S, Reeves JD, Zolla-Pazner S, Hoxie JA, Doms RW, Baribaud F. Truncation of the cytoplasmic domain induces exposure of conserved regions in the ectodomain of human immunodeficiency virus type 1 envelope protein. J Virol. 2002;76(6):2683-91.

31. Aguilar HC, Anderson WF, Cannon PM. Cytoplasmic tail of Moloney murine leukemia virus envelope protein influences the conformation of the extracellular domain: implications for mechanism of action of the $\mathrm{R}$ Peptide. JVirol. 2003;77(2):1281-91.

32. Chen J, Kovacs JM, Peng H, Rits-Volloch S, Lu J, Park D, Zablowsky E, Seaman MS, Chen B. HIV-1 ENVELOPE. Effect of the cytoplasmic domain on antigenic characteristics of HIV-1 envelope glycoprotein. Science. 2015;349(6244):191-5.

33. Das S, Banerjee B, Hossain M, Thangamuniyandi M, Dasgupta S, Chongdar $\mathrm{N}$, Kumar GS, Basu G. Characterization of DNA binding property of the HIV-1 host factor and tumor suppressor protein Integrase Interactor 1 (INI1/hSNF5). PLoS ONE. 2013;8(7):e66581.

34. Boscia AL, Akabori K, Benamram Z, Michel JA, Jablin MS, Steckbeck JD, Montelaro RC, Nagle JF, Tristram-Nagle S. Membrane structure correlates to function of LLP2 on the cytoplasmic tail of HIV-1 gp41 protein. Biophys J. 2013;105(3):657-66.

35. Steckbeck JD, Sun C, Sturgeon TJ, Montelaro RC. Detailed topology mapping reveals substantial exposure of the "cytoplasmic" C-terminal tail (CTT) sequences in HIV-1 Env proteins at the cell surface. PLoS ONE. 2013;8(5):e65220.

36. Cleveland SM, Buratti E, Jones TD, North P, Baralle F, McLain L, Mclnerney T, Durrani Z, Dimmock NJ. Immunogenic and antigenic dominance of a nonneutralizing epitope over a highly conserved neutralizing epitope in the gp41 envelope glycoprotein of human immunodeficiency virus type 1: its deletion leads to a strong neutralizing response. Virology. 2000;266(1):66-78.

37. Blattner C, Lee JH, Sliepen K, Derking R, Falkowska E, de la Pena AT, Cupo A, Julien JP, van Gils M, Lee PS, et al. Structural delineation of a quaternary, cleavage-dependent epitope at the gp41-gp120 interface on intact HIV-1 Env trimers. Immunity. 2014;40(5):669-80.

38. Berlioz-Torrent C, Shacklett BL, Erdtmann L, Delamarre L, Bouchaert I, Sonigo P, Dokhelar MC, Benarous R. Interactions of the cytoplasmic domains of human and simian retroviral transmembrane proteins with components of the clathrin adaptor complexes modulate intracellular and cell surface expression of envelope glycoproteins. J Virol. 1999;73(2):1350-61.

39. Ye L, Bu Z, Vzorov A, Taylor D, Compans RW, Yang C. Surface stability and immunogenicity of the human immunodeficiency virus envelope glycoprotein: role of the cytoplasmic domain. J Virol. 2004;78(24):13409-19.

40. Wei X, Decker JM, Liu H, Zhang Z, Arani RB, Kilby JM, Saag MS, Wu X, Shaw GM, Kappes JC. Emergence of resistant human immunodeficiency virus type 1 in patients receiving fusion inhibitor (T-20) monotherapy. Antimicrob Agents Chemother. 2002;46(6):1896-905.

41. White TA, Bartesaghi A, Borgnia MJ, Meyerson JR, de la Cruz MJ, Bess JW, Nandwani R, Hoxie JA, Lifson JD, Milne JL, et al. Molecular architectures of trimeric SIV and HIV-1 envelope glycoproteins on intact viruses: strain-dependent variation in quaternary structure. PLoS Pathog. 2010;6(12):e1001249.

42. Steckbeck JD, Kuhlmann AS, Montelaro RC. C-terminal tail of human immunodeficiency virus gp41: functionally rich and structurally enigmatic. J Gen Virol. 2013;94(Pt 1):1-19.

43. Bultmann A, Muranyi W, Seed B, Haas J. Identification of two sequences in the cytoplasmic tail of the human immunodeficiency virus type 1 envelope glycoprotein that inhibit cell surface expression. J Virol. 2001;75(11):5263-76.

44. Checkley MA, Luttge BG, Freed EO. HIV-1 envelope glycoprotein biosynthesis, trafficking, and incorporation. J Mol Biol. 2011;410(4):582-608.

45. Zingler K, Littman DR. Truncation of the cytoplasmic domain of the simian immunodeficiency virus envelope glycoprotein increases env incorporation into particles and fusogenicity and infectivity. J Virol. 1993;67(5):2824-31.

46. Freed EO, Myers DJ, Risser R. Mutational analysis of the cleavage sequence of the human immunodeficiency virus type 1 envelope glycoprotein precursor gp160. J Virol. 1989;63(11):4670-5. 
47. Kalia V, Sarkar S, Gupta P, Montelaro RC. Rational site-directed mutations of the LLP-1 and LLP-2 lentivirus lytic peptide domains in the intracytoplasmic tail of human immunodeficiency virus type $1 \mathrm{gp} 41$ indicate common functions in cell-cell fusion but distinct roles in virion envelope incorporation. J Virol. 2003;77(6):3634-46.

48. Steckbeck JD, Sun C, Sturgeon TJ, Montelaro RC. Topology of the C-terminal tail of HIV-1 gp41: differential exposure of the Kennedy epitope on cell and viral membranes. PLoS ONE. 2010;5(12):e15261.

49. Murphy RE, Samal AB, Vlach J, Saad JS. Solution structure and membrane interaction of the cytoplasmic tail of HIV-1 gp41 protein. Structure. 2017;25(11):1708-18.
50. Murakami T, Freed EO. The long cytoplasmic tail of gp41 is required in a cell type-dependent manner for HIV-1 envelope glycoprotein incorporation into virions. Proc Natl Acad Sci USA. 2000;97(1):343-8.

51. Ringe $\mathrm{R}$, Thakar $\mathrm{M}$, Bhattacharya J. Variations in autologous neutralization and CD4 dependence of b12 resistant HIV-1 clade C env clones obtained at different time points from antiretroviral naive Indian patients with recent infection. Retrovirology. 2010;7:76.

52. Montefiori DC, Roederer M, Morris L, Seaman MS. Neutralization tiers of HIV-1. Curr Opin HIV AIDS. 2018;13(2):128-36.
Ready to submit your research? Choose BMC and benefit from:

- fast, convenient online submission

- thorough peer review by experienced researchers in your field

- rapid publication on acceptance

- support for research data, including large and complex data types

- gold Open Access which fosters wider collaboration and increased citations

- maximum visibility for your research: over $100 \mathrm{M}$ website views per year

At BMC, research is always in progress.

Learn more biomedcentral.com/submissions 